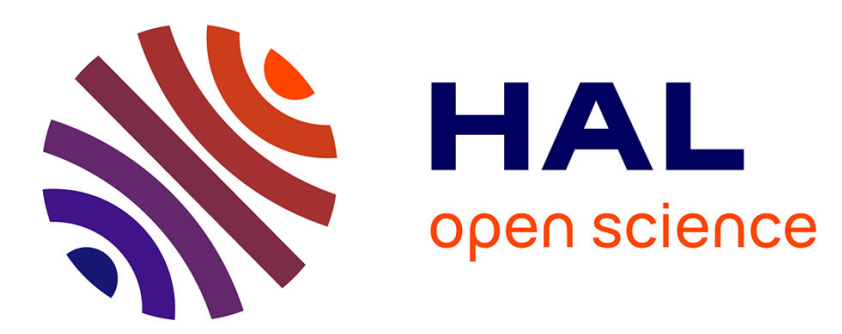

\title{
Periodicity in optimal hierarchical checkpointing schemes for adjoint computations
}

Guillaume Aupy, Julien Herrmann

\section{To cite this version:}

Guillaume Aupy, Julien Herrmann. Periodicity in optimal hierarchical checkpointing schemes for adjoint computations. Optimization Methods and Software, 2017, 32 (3), pp.594-624. 10.1080/10556788.2016.1230612 . hal-01654632

\section{HAL Id: hal-01654632 \\ https://hal.inria.fr/hal-01654632}

Submitted on 4 Dec 2017

HAL is a multi-disciplinary open access archive for the deposit and dissemination of scientific research documents, whether they are published or not. The documents may come from teaching and research institutions in France or abroad, or from public or private research centers.
L'archive ouverte pluridisciplinaire HAL, est destinée au dépôt et à la diffusion de documents scientifiques de niveau recherche, publiés ou non, émanant des établissements d'enseignement et de recherche français ou étrangers, des laboratoires publics ou privés. 
To appear in Optimization Methods \& Software

Vol. 00, No. 00, Month 20XX, 1-34

\title{
ORIGINAL RESEARCH
}

\section{Periodicity in optimal hierarchical checkpointing schemes for adjoint computations}

\author{
Guillaume Aupy ${ }^{\ddagger b *}$ and Julien Herrmann ${ }^{\dagger b}$ \\ $\ddagger$ Vanderbilt University, Nashville TN, USA \\ $\dagger$ Georgia Institute of Technology, Atlanta GA, USA
}

(January 2016)

\begin{abstract}
We reexamine the work of Aupy et al. on optimal algorithms for hierarchical adjoint computations, where two levels of memories are available. The previous optimal algorithm had a quadratic execution time. Here, with structural arguments, namely periodicity, on the optimal solution, we provide an optimal algorithm in constant time and space, with appropriate pre-processing. We also provide an asymptotically optimal algorithm for the online problem, when the adjoint chain size is not known before-hand. Again, these algorithms rely on the proof that the optimal solution for hierarchical adjoint computations is weakly periodic. We conjecture a closed-form formula for the period. Finally, we assess the convergence speed of the approximation ratio for the online problem through simulations.
\end{abstract}

Keywords: Optimal algorithms, Online, Asymptotic optimality, Checkpointing, Adjoint computation, Program reversal, Automatic differentiation

AMS Subject Classification: 65Y20, 68W40, 49N45, 90C30

This work was supported by the French Research Agency (ANR) through the Rescue project; and the Inria-Illinois-ANL-BSC-JSC-Riken/AICS Joint Laboratory for ExtremeScale Computing.

\footnotetext{
${ }^{b}$ Part of this work was done while the authors were at LIP - ENS Lyon, INRIA, CNRS, Université Lyon 1, France

*Corresponding author. Email: guillaume.aupy@vanderbilt.edu.
} 


\section{Introduction}

The need to efficiently compute the gradient to a cost function arises frequently in many areas of scientific computing, including mathematical optimization, uncertainty quantification, and nonlinear systems of equations. In gradient based optimization, discrete adjoint methods are widely used for the gradient computation when the problem possesses an extensive amount of design variables. These discrete adjoint methods consist in solving a set of governing equations forward and then solving the adjoint problem backwards in time in order to obtain the adjoint variables. In the case of nonlinear governing equations, there is a need to keep track of every intermediate results during the computation of the forward solution in order to be able to integrate the corresponding adjoint differential equation backward. In general, intermediate function values must be stored or recomputed when needed [2]. A popular storage or recomputation strategy for functions that have some sort of natural "time step" is to save (checkpoint) the state at each time step during the function computation (forward sweep) and use this saved state in the derivative computation (reverse sweep). If the storage is inadequate for all states, one can checkpoint only some states and recompute the unsaved states as needed. Thus, there is a need to find a good compromise between memory consumption and runtime overhead due to recomputations. The checkpointing scheme in the literature can be split in two categories: the ones dealing with an offline framework, and the ones dealing with an online framework:

- If the number of time step in the forward sweep is known a priori (offline framework), Griewank and Walther proved that, given a fixed number of checkpoints, the schedule that minimizes the amount of recomputation is a binomial checkpointing strategy [3, 4]. They also gave a closed formula to compute the indices of the forward step to store in the memory [10]. Although the number of checkpoint reads and writes is taken into account as a secondary objective, the problem model they used implicitly assumes that reading and writing checkpoints are free, but the number of available checkpoint is limited (one memory framework). Later, Stumm and Walther [8] consider the case where a second type of storage is available: the checkpoints can also be written onto a disk with an unlimited storage capacity but whose time to read or write a checkpoint can no longer be ignored (hierarchical memory framework). They designed a hierarchical heuristic using a binomial checkpointing strategy based on the optimal algorithm for the case without disk. Despite its good experimental results, the heuristic is sub-optimal for the hierarchical memory framework. More recently, Aupy et al. [1] provided a polynomial time algorithm for determining the optimal schedule using both memory and disk, if the number of time step is known a priori.

- However, in the context of flow control, the partial differential equations to be solved are usually stiff, and the solution process relies therefore on some adaptive time stepping procedure. Hence, the number of time steps performed is known only after the complete integration (online framework). One has to decide on the fly during the forward sweep where to place the checkpoints without knowing how many time steps are left to perform. Hinze and Sternberg [5] proposed a heuristic to generate online schedules as long as the number of time-steps does not exceed a given upper bound. This heuristic is designed for the one memory framework and relies on a dynamic rearrangement of the checkpoints. Later, Stumm and Walther [9] designed a new algorithm to compute optimal online checkpointing schedules (i) when the number of time step does not exceed a 
(larger) upper bound, and (ii) the repetition number is equal to 2. They also give asymptotic optimal solution when the number of time steps is large and the repetition number equal to 3. For larger adjoint computations, Wang and al. [11] provided a dynamic online algorithm that minimizes the maximum number of recomputation for a single forward step, matching the optimal offline repetition number and ensuring that the overall computational cost has a theoretical upper bound. To the best of our knowledge, there is no previous theoretical study on online hierarchical adjoint computations, since no optimal algorithm for the offline hierarchical memory framework were known before [1 bridged that gap. This paper aims to extend their results to the online framework.

In this paper, we provide a theoretical analysis of the optimal offline hierarchical algorithm proposed by Aupy et al. [1, which allows us to reduce significantly its computation time and space (from quadratic to constant). This optimization relies on the proof that for any adjoint chain size, there exists an optimal disk checkpointing strategy that is weakly periodic, which means that all the intervals between two consecutive disk checkpoints are of the same size except for a bounded number of them. This bound and the optimal interval only depend on the architecture parameters, namely the number of available memory checkpoints and the time to read or write a checkpoint on the disk. These observations allow us to provide in this paper two valuable contributions. First, we can design an optimal algorithm in constant time and space (independent of the adjoint chain size) for the offline hierarchical memory framework, with an appropriate pre-processing depending on the architecture parameters. This represents a major improvement compared to the computational expensive algorithm provided by Aupy et al. [1. Second, we can design an asymptotically optimal algorithm for the online hierarchical memory framework bridging that gap in the literature.

The rest of this paper is organized as follows. Section 2 lays the grounds of hierarchical adjoint computation and recalls important results from [1]. Section 3 introduces notations that will be used during the paper. Section 4 constitutes the heart of this paper and provides many structural arguments on the optimal hierarchical schedules. These results are used in Section 5 to design the asymptotically optimal algorithm for the online hierarchical framework. Finally, Section 6 assess the performance of the asymptotically optimal online algorithm compared to the actual optimal algorithm that can be computed when the actual size of the adjoint chain is known beforehand.

\section{Framework}

\subsection{The Adjoint Computation problem}

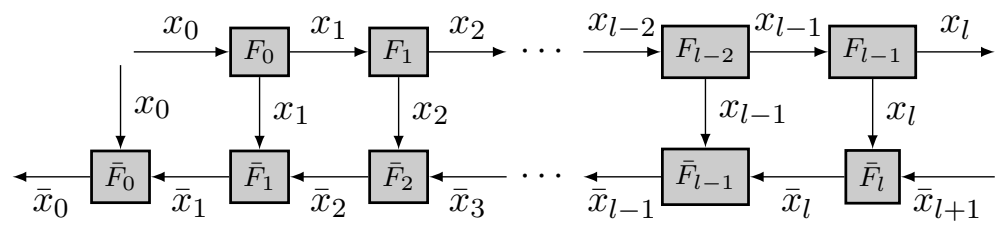

Figure 1.: The data dependencies in the Adjoint Computation chain.

Definition 1 (Adjoint Computation [4, 8]) An adjoint computation with $l$ time steps 
can be described by the following set of equations:

$$
\begin{gathered}
F_{i}\left(x_{i}\right)=x_{i+1} \text { for } 0 \leq i<l \\
\bar{F}_{i}\left(x_{i}, \bar{x}_{i+1}\right)=\bar{x}_{i} \text { for } 0 \leq i \leq l
\end{gathered}
$$

The dependencies between these operation $\mathrm{S}^{1}$ are represented by the graph $\mathcal{G}=(V, E)$ depicted in Figure 1.

The $F$ computations are called forward steps. They have an execution cost of $u_{f} \in \mathbb{R}^{+}$. The $\bar{F}$ computations are called backward steps, they have an execution cost of $u_{b} \in \mathbb{R}^{+}$. If $\bar{x}_{l+!}$ is initialized appropriately, then at the conclusion of the adjoint computation, $\bar{x}_{0}$ will contain the gradient with respect to the initial state $\left(x_{0}\right)$.

DEFinition 2 (Platform) We consider a platform with three storage locations:

- Buffers: there are two buffers, the top buffer and the bottom buffer. The top buffer is used to store a value $x_{i}$ for some $i$, while the bottom buffer is used to store a value $\bar{x}_{i}$ for some $i$. For a computation $(F$ or $\bar{F})$ to be executed, its input values have to be stored in the buffers. Let $\mathcal{B}^{\top}$ and $\mathcal{B}^{\perp}$ denote the content of the top and bottom buffers. In order to start the execution of the chain, $x_{0}$ must be stored in the top buffer, and $\bar{x}_{l+1}$ in the bottom buffer. Hence without loss of generality, we assume that at the beginning of the execution, $\mathcal{B}^{\top}=\left\{x_{0}\right\}$ and $\mathcal{B}^{\perp}=\left\{\bar{x}_{l+1}\right\}$.

- Memory: there are $c_{m}$ slots of memory where the content of a buffer can be stored. The time to write from buffer to memory is $w_{m}$. The time to read from memory to buffer is $r_{m}$. Let $\mathcal{M}$ be the set of $x_{i}$ and $\bar{x}_{i}$ values stored in the memory. The memory is empty at the beginning of the execution $(\mathcal{M}=\emptyset)$.

- Disks: there are $c_{d}$ slots of disks where the content of a buffer can be stored. The time to write from buffer to disk is $w_{d} \in \mathbb{R}^{+}$. The time to read from disk to buffer is $r_{d} \in \mathbb{R}^{+}$. Let $\mathcal{D}$ be the set of $x_{i}$ and $\bar{x}_{i}$ values stored in the disk. The disk is empty at the beginning of the execution $(\mathcal{D}=\emptyset)$.

Memory and disk are generic terms for a two-level storage system, modeling any platform with a dual memory system, including (i) a cheap-to-access first-level memory, of limited size; and (ii) and a costly-to-access second-level memory, whose size is very large in comparison of the first-level memory. The pair (memory, disk) can be replaced by (cache, memory) or (disk, tape) or any relevant hardware combination.

Intuitively, the core of the Adjoint Computation problem is the following: after the execution of a forward step, its output is kept in the top buffer only. If it is not saved in memory or disk before the next forward step, it is lost and will have to be recomputed when needed for the corresponding backward step. When no disk storage is available, the problem is to minimize the number of re-computations in the presence of limited (but free-to-access) memory slots. When disk storage is added, the problem becomes even more challenging: saving data on disk can save some recomputations, and a trade-off must be found between the cost of disk accesses and that of recomputations. In accordance to the scheduling literature, we use the term makespan for the total execution time.

Here, we consider the problem with an unlimited number of disk storage, where, while reading and writing from/to memory is still free, reading and writing from/to disk has a cost. We assume that at the beginning of the execution, both the memory and the disk

\footnotetext{
${ }^{1}$ In the original approach by Griewank [3], an extra $F_{l}$ operation was included. It is not difficult to take this extra operation into account.
} 


\begin{tabular}{|c|c|c|c|}
\hline & Operation & Input & Action \\
\hline$F_{i}$ & $\begin{array}{l}\text { Executes one forward step } F_{i}(\text { for } i \in\{0, \ldots, l-1\}) \text {. } \\
\text { This operation takes a time } \operatorname{cost}\left(F_{i}\right)=u_{f}\end{array}$ & $\mathcal{B}^{\top}=\left\{x_{i}\right\}$ & $\mathcal{B}^{\top} \leftarrow\left\{x_{i+1}\right\}$ \\
\hline$F_{i \rightarrow i^{\prime}}$ & Denotes the sequence $F_{i} \cdot F_{i+1} \cdot \ldots \cdot F_{i^{\prime}}{ }^{1}$. & $\mathcal{B}^{\top}=\left\{x_{i}\right\}$ & $\mathcal{B}^{\top} \leftarrow\left\{x_{i^{\prime}}\right\}$ \\
\hline $\bar{F}_{i}$ & $\begin{array}{l}\text { Executes the backward } \operatorname{step} \bar{F}_{i}(i \in\{0, \ldots, l\}) \text {. This } \\
\text { operation takes a time } \operatorname{cost}\left(\bar{F}_{i}\right)=u_{b} \text {. }\end{array}$ & $\begin{array}{l}\mathcal{B}^{\top}=\left\{x_{i}\right\} \\
\mathcal{B}^{\perp}=\left\{\bar{x}_{i+1}\right\}\end{array}$ & $\mathcal{B}^{\perp} \leftarrow\left\{\bar{x}_{i}\right\}$ \\
\hline$W_{i}^{m}$ & $\begin{array}{l}\text { Writes the value } x_{i} \text { of the top buffer into the memory. } \\
\text { This operation takes time } \operatorname{cost}\left(W_{i}^{m}\right)=w_{m} \text {. }\end{array}$ & $\mathcal{B}^{\top}=\left\{x_{i}\right\}$ & $\mathcal{M} \leftarrow \mathcal{M} \cup\left\{x_{i}\right\}^{2}$ \\
\hline$R_{i}^{m}$ & $\begin{array}{l}\text { Reads the value } x_{i} \text { in the memory, and puts it into the } \\
\text { top buffer. This operation takes a time } \operatorname{cost}\left(R_{i}^{m}\right)=r_{m} \text {. }\end{array}$ & $x_{i} \in \mathcal{M}$ & $\mathcal{B}^{\top} \leftarrow\left\{x_{i}\right\}$ \\
\hline$W_{i}^{d}$ & $\begin{array}{l}\text { Writes the value } x_{i} \text { of the top buffer into the disk. This } \\
\text { operation takes a time } \operatorname{cost}\left(W_{i}^{d}\right)=w_{d} \text {. }\end{array}$ & $\mathcal{B}^{\top}=\left\{x_{i}\right\}$ & $\mathcal{D} \leftarrow \mathcal{D} \cup\left\{x_{i}\right\}$ \\
\hline$R_{i}^{d}$ & $\begin{array}{l}\text { Reads the value } x_{i} \text { in the disk and puts it into the top } \\
\text { buffer. This operation takes a time } \operatorname{cost}\left(R_{i}^{d}\right)=r_{d} \text {. }\end{array}$ & $x_{i} \in \mathcal{D}$ & $\mathcal{B}^{\top} \leftarrow\left\{x_{i}\right\}$ \\
\hline
\end{tabular}

Table 1.: Operations performed by a schedule.

are empty:

Problem $1\left(\operatorname{ProB}_{\infty}\left(l, c_{m}, w_{d}, r_{d}\right)\right)$ We want to minimize the makespan of the Adjoint Computation problem with the following parameters:

\begin{tabular}{|r|l|l|}
\hline & & Initial state: \\
\hline Adjoint Computation chain: & size $l$ & \\
Steps: & $u_{f}, u_{b}$ & \\
Memory: & $c_{m}, w_{m}=r_{m}=0$ & $\mathcal{M}=\emptyset$ \\
Disks: & $c_{d}=+\infty, w_{d}, r_{d}$ & $\mathcal{D}=\emptyset$ \\
Buffers: & $\mathcal{B}^{\top}, \mathcal{B}^{\perp}$ & $\mathcal{B}^{\top}=\left\{x_{0}\right\}, \mathcal{B}^{\perp}=\left\{\bar{x}_{l+1}\right\}$ \\
\hline
\end{tabular}

\section{$2.2 \quad$ Summary of our previous work}

In our previous work [1, we were able to compute in polynomial time an optimal solution to $\operatorname{ProB}_{\infty}\left(l, c_{m}, w_{d}, r_{d}\right)$ : Disk-Revolve. An optimal solution to $\operatorname{ProB}_{\infty}\left(l, c_{m}, w_{d}, r_{d}\right)$ is a sequence of operations from Table 1 that executes an Adjoint Computation chain of size $l$ in minimal time.

Definition 3 For every Adjoint Computation problem, we use these definitions:

- (Valid schedule) A valid schedule is a sequence of operations from Table 1 such that all operations of the schedule are valid (they respect the input constraints).

- (Feasible solution) A feasible solution to a given Adjoint Computation problem (such as $\operatorname{PROB}_{\infty}\left(l, c_{m}, w_{d}, r_{d}\right)$ ) is a valid schedule for the initial states of the buffers described by the problem, that returns the value $\overline{x_{0}}$ in the bottom buffer

\footnotetext{
${ }^{1}$ Here, and in the rest of the paper, for any operations $X_{1}$ and $X_{2}$, the sequence $X_{1} \cdot X_{2}$ represents the sequential execution of operation $X_{1}$ and operation $X_{2}$ in this order.

${ }^{2}$ Assuming $|\mathcal{M}|<c_{m}$, or $x_{i} \in \mathcal{M}$.
} 
at the end of the execution.

- (Optimal solution) An optimal solution to a given Adjoint Computation problem is a solution such that there is no solution with a strictly smaller makespan.

DeFinition $4\left(\mathrm{Opt}_{\infty}\left(l, c_{m}, w_{d}, r_{d}\right)\right)$

Given $l \in \mathbb{N}, c_{m} \in \mathbb{N}, w_{d} \in \mathbb{R}^{+}$and $r_{d} \in \mathbb{R}^{+}$, $\operatorname{Opt}_{\infty}\left(l, c_{m}, w_{d}, r_{d}\right)$ is the execution time of an optimal solution to $\operatorname{PROB}_{\infty}\left(l, c_{m}, w_{d}, r_{d}\right)$.

In our previous work [1, we solved $\operatorname{PROB}_{\infty}\left(l, c_{m}, w_{d}, r_{d}\right)$ by reducing it to another problem: $\overline{\mathrm{PROB}}_{1}^{(d)}\left(l, c_{m}, r_{d}\right)$ (see Problem 2). We defined $\mathrm{Opt}_{1}^{(d)}\left(l, c_{m}, r_{d}\right)$ as the execution time of an optimal solution to $\overline{\mathrm{PROB}}_{1}^{(d)}\left(l, c_{m}, r_{d}\right)$.

Problem $2\left(\overline{\operatorname{PrOB}}_{1}^{(d)}\left(l, c_{m}, r_{d}\right)\right)$ We want to minimize the makespan of the Adjoint Computation problem with the following parameters.

\begin{tabular}{|r|l|l|}
\hline & & Initial state: \\
\hline Adjoint Computation chain: & size $l$ & \\
Steps: & $u_{f}, u_{b}$ & \\
Memory: & $c_{m}, w_{m}=r_{m}=0$ & $\mathcal{M}=\emptyset$ \\
Disks: & $c_{d}=1, w_{d}=+\infty, r_{d}$ & $\mathcal{D}=\left\{x_{0}\right\}$ \\
Buffers: & $\mathcal{B}^{\top}, \mathcal{B}^{\perp}$ & $\mathcal{B}^{\top}=\left\{x_{0}\right\}, \mathcal{B}^{\perp}=\left\{\bar{x}_{l+1}\right\}$ \\
\hline
\end{tabular}

Because details of this instance are unimportant for this paper and would complicate the reading process, we refer the interested reader to our previous work (Definition 3.13, [1]) for those details. The algorithm solving this problem uses the binomial checkpointing algorithm Revolve, designed by Griewank and Walther [4, that solves the problem with only memory and no disk (REVOLVE $\left(l, c_{m}\right)$ returns an optimal solution sequence to Problem 3 Prob $\left(l, c_{m}\right)$ below). We denote $\operatorname{Opt}_{0}\left(l, c_{m}\right)$ the execution time of REVOLVE.

The main result of our previous work [1] is the dynamic programs to compute the value of $\mathrm{Opt}_{1}^{(d)}\left(l, c_{m}, r_{d}\right)$ and $\mathrm{Opt}_{\infty}\left(l, c_{m}, w_{d}, r_{d}\right)$ (see Theorem 1).

Theorem 1 (Theorems 1 and 3, 1]) Let $l \in \mathbb{N}, c_{m} \in \mathbb{N}, w_{d} \in \mathbb{R}^{+}$and $r_{d} \in \mathbb{R}^{+}$:

$$
\begin{gathered}
O p t_{\infty}\left(l, c_{m}, w_{d}, r_{d}\right)=\min \left\{\begin{array}{l}
O p t_{0}\left(l, c_{m}\right) \\
w_{d}+\min _{1 \leq j \leq l-1}\left\{j u_{f}+O p t_{\infty}\left(l-j, c_{m}, w_{d}, r_{d}\right)+r_{d}+O p t_{1}^{(d)}\left(j-1, c_{m}, r_{d}\right)\right\}
\end{array}\right. \\
O p t_{1}^{(d)}\left(l, c_{m}, r_{d}\right)=\min \left\{\begin{array}{l}
O p t_{0}\left(l, c_{m}\right) \\
\min _{1 \leq j \leq l-1}\left\{j u_{f}+O p t_{0}\left(l-j, c_{m}\right)+r_{d}+O p t_{1}^{(d)}\left(j-1, c_{m}, r_{d}\right)\right\}
\end{array}\right.
\end{gathered}
$$

Based on these dynamic programs, we designed a polynomial algorithm 1D-REvolve that, given the values $l, c_{m}$ and $r_{d}$ returns $1 \mathrm{D}$-Revolve $\left(l, c_{m}, r_{d}\right)$, an optimal sequence for $\overline{\operatorname{PROB}}_{1}^{(d)}\left(l, c_{m}, r_{d}\right)$.

Problem $3\left(\operatorname{Prob}\left(l, c_{m}\right)\right)$ We want to minimize the makespan of the Adjoint Computation problem with the following parameters: 


\begin{tabular}{|r|l|l|}
\hline & & Initial state: \\
\hline Adjoint Computation chain: & size $l$ & \\
Steps: & $u_{f}, u_{b}$ \\
Memory: & $c_{m}, w_{m}=r_{m}=0$ & $\mathcal{M}=\emptyset$ \\
Disks: & $c_{d}=0$ & \\
Buffers: & $\mathcal{B}^{\top}, \mathcal{B}^{\perp}$ & $\mathcal{B}^{\top}=\left\{x_{0}\right\}, \mathcal{B}^{\perp}=\left\{\bar{x}_{l+1}\right\}$ \\
\hline
\end{tabular}

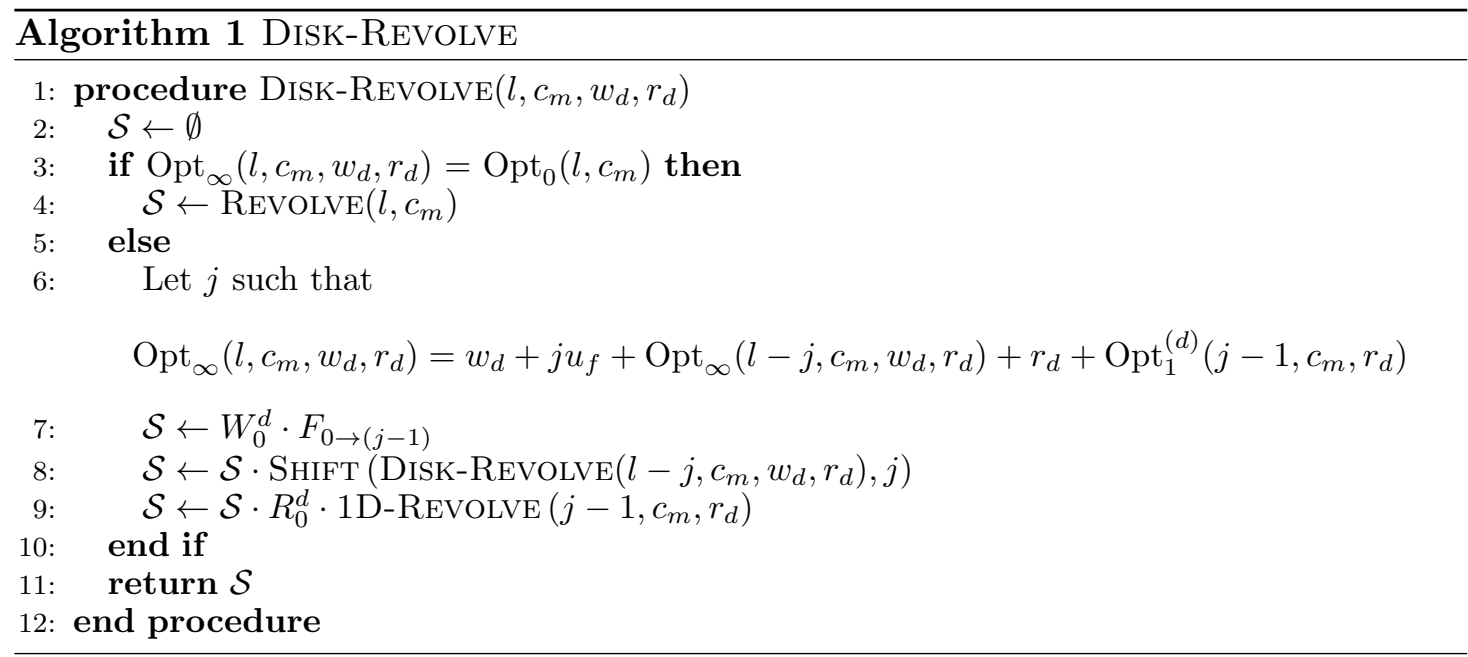

We also defined SHIFT, the routine that takes a sequence $S$ and an index ind and returns $S$ shifted by ind (meaning for all $i \leq l, s \in\{m, d\}, W_{i}^{s}$ are replaced by $W_{i+\text { ind }}^{s}, R_{i}^{s}$ are replaced by $R_{i+\text { ind }}^{s}, F_{i}$ by $F_{i+\text { ind }}$, and $\bar{F}_{i}$ by $\left.\bar{F}_{i+\text { ind }}\right)$. Note that sequence SHIFT $(S$, ind) has the same execution time as sequence $S$. Finally, we designed algorithm Disk-REvolve (Algorithm 1) that, given an adjoint computation chain of size $l \in \mathbb{N}$, $c_{m} \in \mathbb{N}$ memory slots, a cost $w_{d} \geq 0$ to write to disk and a cost $r_{d} \geq 0$ to read from disk, returns Disk-Revolve $\left(l, c_{m}, w_{d}, r_{d}\right)$ an optimal schedule for $\operatorname{PROB}_{\infty}\left(l, c_{m}, w_{d}, r_{d}\right)$. The time complexity of this algorithm is $O\left(l^{2}\right)$.

\section{Dominant Sequences and Dominant Optimal Sequences}

In this paper, we propose a different algorithm to compute optimal solutions to $\operatorname{ProB}_{\infty}\left(l, c_{m}, w_{d}, r_{d}\right)$. We introduce a family of sequences that we call Dominant Sequences (DS). A Dominant Sequence is a sequence that can be returned by algorithm AlgoDom (Algorithm 2). They are entirely characterised by a tuple $\left(m_{1}, \cdots, m_{n_{l}}\right.$; res), the arguments of AlgODom. We call these sequences dominant, because we show in Section 4.3 that for every values $l, c_{m}, r_{d}$ and $w_{d}$, there is a Dominant Sequence that is optimal for $\operatorname{PROB}_{\infty}\left(l, c_{m}, w_{d}, r_{d}\right)$. Such a sequence is called a Dominant Optimal Sequence (DOS).

To begin, we show that when $l=\sum_{i=1}^{n_{l}} m_{i}+$ res the sequence returned by $\operatorname{AlgoDom}\left(m_{1}, \cdots, m_{n_{l}} ;\right.$ res $)$ indeed returns a feasible solution to $\operatorname{PROB}_{\infty}\left(l, c_{m}, w_{d}, r_{d}\right)$.

Lemma 1 Let $\left(m_{1}, \cdots, m_{n_{l}}\right.$, res $) \in \mathbb{N}^{n_{l}+1}$ such that $l=\sum_{i=1}^{n_{l}} m_{i}+$ res, then $\operatorname{AlgoDom}\left(m_{1}, \cdots, m_{n_{l}} ;\right.$ res $)$ is a feasible solution for $\mathrm{PROB}_{\infty}\left(l, c_{m}, w_{d}, r_{d}\right)$.

Proof. In order to prove that AlgoDom $\left(m_{1}, \cdots, m_{n_{l}} ;\right.$ res $)$ is a feasible solution, we need to show that each operation done is valid. 


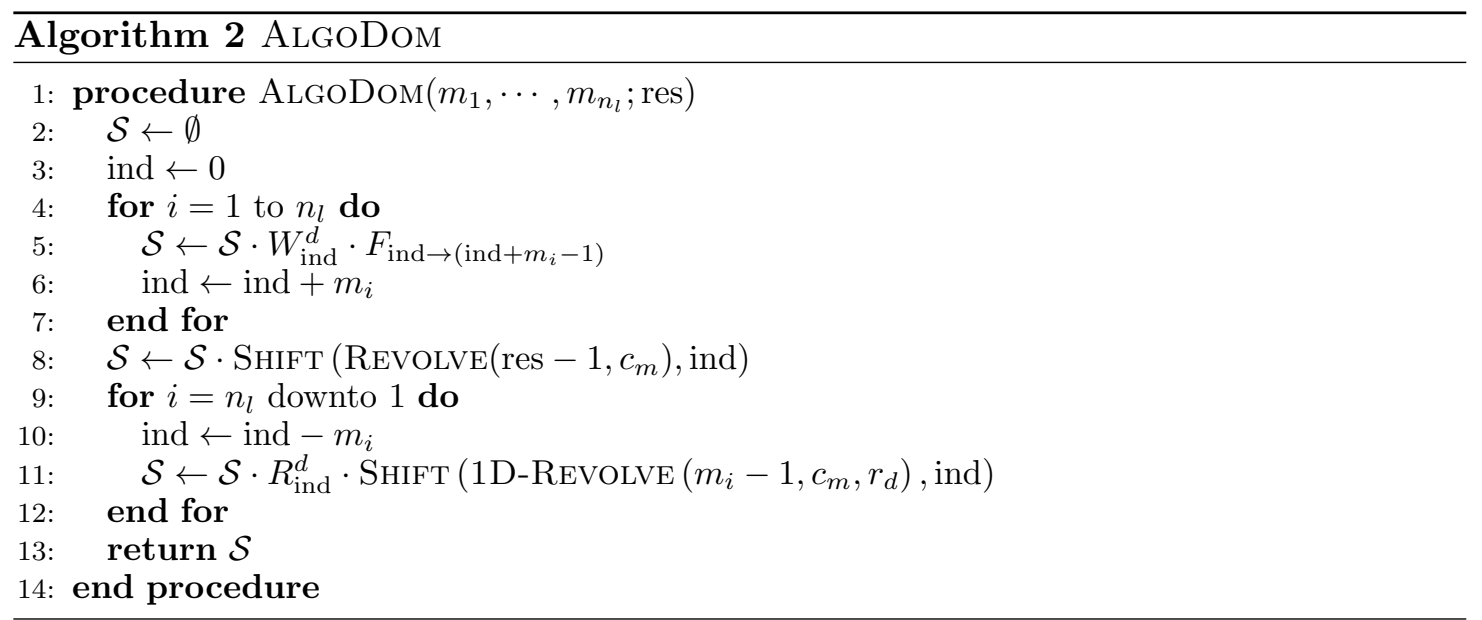

- The operations introduced by the "for loop" on line 4 are valid as each $F$-operation is consecutive (so the needed information is in the top-buffer), and the $W_{\text {ind }}^{d}$ operations immediately follow the $F_{\text {ind-1 }}$ that puts the value $x_{\text {ind }}$ in the top buffer ( $W_{0}^{d}$ is also valid as it is the first operation and the value $x_{0}$ is in the top buffer initially).

- We know that Revolve(res $\left.-1, c_{m}\right)$ returns a valid sequence for an Adjoint Computation chain of size res -1 as long as the value $x_{0}$ is in the top buffer and $\bar{x}_{\text {res }+1}$ is in the bottom buffer. Function $\operatorname{ShIFT}(\cdot$, ind $)$ shifts all the indexes by ind. Then, since at the end of the first "for loop" ind $=l-$ res, we can state that applying the sequence $\operatorname{Shift}\left(\operatorname{Revolve}\left(\right.\right.$ res $\left.-1, c_{m}\right)$, ind) on line 8 is valid if the value $x_{\text {ind }}=x_{l-\text { res }}$ is in the top buffer and the value $\bar{x}_{\text {res }+1+\text { ind }}=\bar{x}_{l+1}$ is in the bottom buffer, which is true after the first "for loop".

- We show by induction that the second "for loop" on line9 is correct. We use as induction hypotheses $H(i)$ : at the beginning of the $i^{\text {th }}$ iteration, $\bar{x}_{\sum_{j=1}^{i} m_{j}}$ is in the bottom buffer and $H^{\prime}(i)$ : iteration $i$ is correct.

At the end of sequence Revolve(res $-1, c_{m}$ ), the value $\bar{x}_{0}$ is in the bottom buffer, which means that after sequence Shift (REvolve(res $\left.-1, c_{m}\right)$, ind), the value $\bar{x}_{\text {ind }}=\bar{x}_{l-\text { res }}=\bar{x}_{\sum_{j=1}^{n_{l}} m_{j}}$. Thus $H\left(n_{l}\right)$ is true. Assume it is true until the $i^{\text {th }}$ iteration. Then at the beginning of the $i^{\text {th }}$ iteration, ind $=\sum_{j=1}^{i} m_{j}$ and $x_{\text {ind }}$ was indeed written on disk during the $i^{\text {th }}$ iteration of the "for loop" on line 4 1D-Revolve is possible because $\mathrm{Opt}_{1}^{(d)}\left(m_{i}-1, c_{m}, r_{d}\right)$ takes as input $x_{0}$ in the top buffer and stored on disk, and $\bar{x}_{m_{i}}$ in the top buffer. Hence showing $H^{\prime}(i)$. Furthermore, the result is then: $\bar{x}_{\text {ind }}=\bar{x}_{\sum_{j=1}^{i} m_{j}}$ in the bottom buffer, which shows $H(i-1)$.

The result of the last iteration of the "for loop" on line 9 is then exactly $\bar{x}_{0}$, which shows the correctness of AlgoDom.

We now formally define notations that are used throughout this paper.

Definition 5 Let $l \geq 0, n_{l} \geq 0$, and $m_{1}, \cdots, m_{n_{l}}$, res such that $l=\sum_{i=1}^{n_{l}} m_{i}+$ res, then

- We shorten the sequence of operation returned by $\operatorname{AlgoDom}\left(m_{1}, \cdots, m_{n_{l}}\right.$; res $)$ by $\mathcal{S}=\left(m_{1}, \cdots, m_{n_{l}} ;\right.$ res $)\left(\mathcal{S}=(; l)\right.$ if $\left.n_{l}=0\right)$. $\mathcal{S}$ is called a Dominant Sequence (DS) for $\operatorname{PROB}_{\infty}\left(l, c_{m}, w_{d}, r_{d}\right)$.

- $\mathcal{E} x e c(\mathcal{S})$ is the cost (or the execution time) of the sequence $\mathcal{S}$, that is to say the 


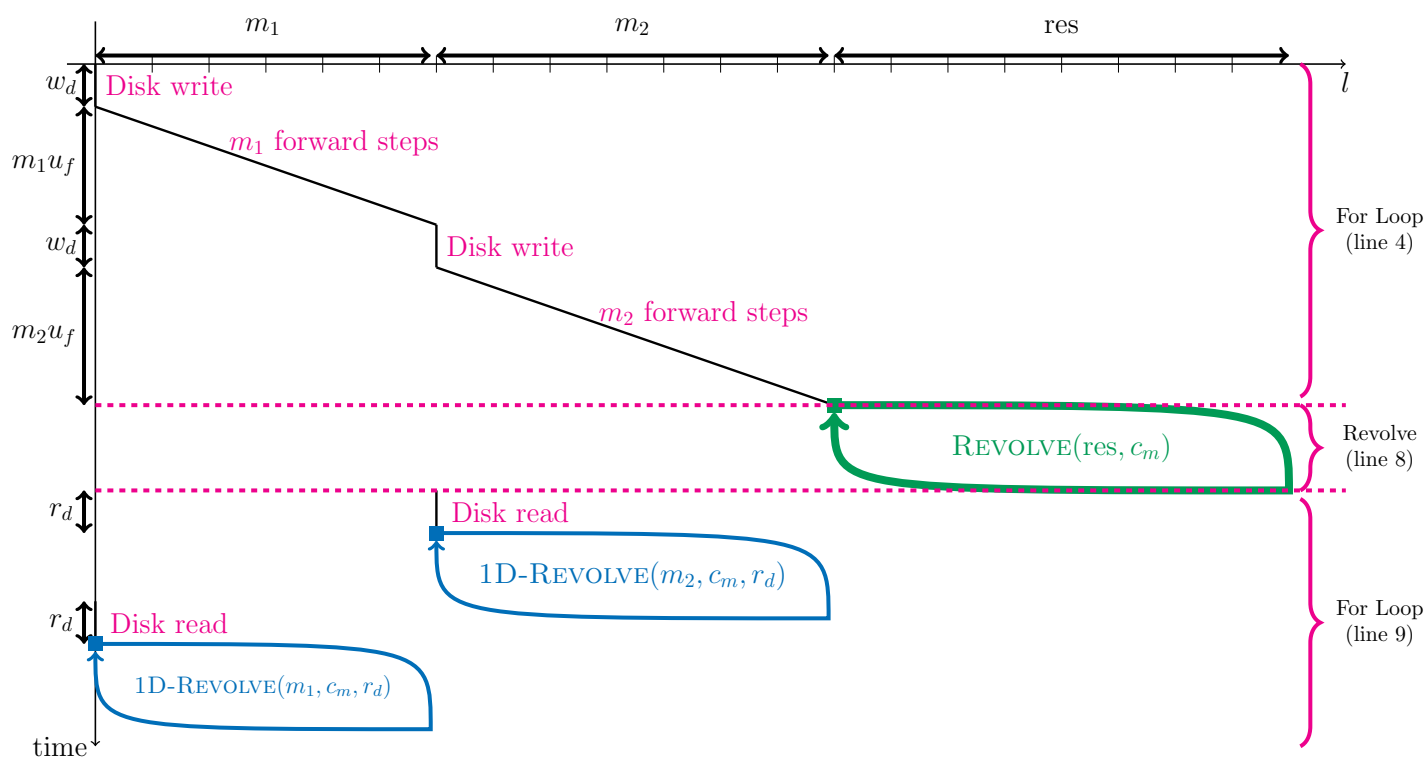

Figure 2.: Example of execution of $\operatorname{AlgoDom}\left(m_{1}, m_{2} ;\right.$ res $)$

sum of all the operations cost in $\mathcal{S}$.

- $\mathcal{S}=\left(m_{1}, \cdots, m_{n_{l}} ;\right.$ res $)$ is a Dominant Optimal Sequence (DOS) for $\operatorname{PROB}_{\infty}\left(l, c_{m}, w_{d}, r_{d}\right)$ if the sequence $\mathcal{S}$ is an optimal solution to $\operatorname{PROB}_{\infty}\left(l, c_{m}, w_{d}, r_{d}\right)$, that is to say $\mathcal{E} x e c(\mathcal{S})=\operatorname{Opt}_{\infty}\left(l, c_{m}, w_{d}, r_{d}\right)$

- Let $\mathcal{S}=\left(m_{1}, \cdots, m_{n_{l}} ;\right.$ res $)$ a DS, the $m_{1}, \cdots, m_{n_{l}}$ are called the periods of $\mathcal{S}$, res is called the residual and $n_{l}$ is the number of periods of $\mathcal{S}$.

\section{Periodicity in optimal hierarchical checkpointing schemes}

In this work, we consider a platform with $c_{m}$ memory slots, a writing cost to disk $w_{d}$ and a reading cost from disk $r_{d}$. Throughout the rest of the paper, we denote all these platform parameters by a unique symbol $X=\left(c_{m}, r_{d}, w_{d}\right)$.

The main contribution of this paper is the following theorem:

THEOREM 2 (Weak periodicity) There exists $l_{X}, i_{X}$ and $m_{X}$ such that: for all $l \geq l_{X}$, there exists $n_{l} \geq i_{X}$, and $\left(m_{1}, \cdots, m_{n_{l}}\right.$,res) $\in \mathbb{N}^{n_{l}+1}$ (with $l=\sum_{i=1}^{n_{l}} m_{i}+$ res) such that $\operatorname{AlgoDom}\left(m_{1}, \cdots, m_{n_{l}} ;\right.$ res $)$ is optimal for $\operatorname{PROB}_{\infty}\left(l, c_{m}, w_{d}, r_{d}\right)$ and

$$
\begin{gathered}
\forall i<n_{l}-i_{X}, m_{i}=m_{X} \\
\sum_{i=n_{l}-i_{X}}^{n_{l}} m_{i}+r e s<l_{X}
\end{gathered}
$$

This result says that for every problem size, there exists a Dominant Optimal Sequence such that all the periods $m_{i}$ 's are equal to $m_{X}$, except for a bounded number of them.

This bound, $i_{X}$, only depends on the parameters $X$ of the architecture. Stronger results would be to give values for $i_{X}$ and for $m_{X}$. We derive the value for $m_{X}$ experimentally in Section 6 .

A consequence of this result is: 
COROLlary 1 (Online algorithm) There exists an asymptotically optimal algorithm for the online version (that is when the size of the chain is not known before-hand) of Problem 1 .

The implications of these results is

(1) to provide a constant time and constant space algorithm (presented in Section 6) to compute optimal solutions for the Adjoint Computation offline problem, while previous results took quadratic time and quadratic space complexity.

(2) to provide an asymptotically optimal algorithm for the online problem (presented in Section 5).

In the rest of this section, we prove Theorem 2. In order to do so, we first start by giving structural arguments on the two core algorithms of the optimal solution: REvOLVE and 1D-Revolve in Section 4.1. Then we introduce some properties on the execution time of Dominant Sequences (DS), before showing that for all size $l$ of the Adjoint Computation chain, there exists a Dominant Sequence that is optimal (Theorem 6).

In order to prove Theorem 2 .

(1) We first construct a set of admissible periods $\mathcal{M}_{X}^{(\mathrm{Adm})}$ in Section 4.4 .

(2) Then we show that the admissible periods have a maximum size in Section 4.5 hence showing that the set $\mathcal{M}_{X}^{(\mathrm{Adm})}$ is finite.

(3) We then construct $\mathcal{M}_{X} \subset \mathcal{M}_{X}^{(\text {Adm) }}$ such that for all Adjoint Computation chains, there exists a DOS with periods solely in $\mathcal{M}_{X}$ in Section 4.6 .

(4) Finally, we show the result by contructing $m_{X}$, a period that is dominant over all other periods of $\mathcal{M}_{X}$.

\subsection{Properties of a period}

In this section we give structural arguments on 1D-REvOLVE. In particular we are interested in the number of forward steps done by 1D-REVOLVE before the first memory write.

By definition 1D-Revolve can either behave as Revolve (and not use the disk checkpoint at hand), or do a certain number of disk reads. We discuss properties of the execution times of Revolve $\left(\mathrm{Opt}_{0}\left(l, c_{m}\right)\right)$ in Section 4.1.1 and of 1D-Revolve in general $\left(\mathrm{Opt}_{1}^{(d)}\left(l, c_{m}, r_{d}\right)\right)$ in Section 4.1 .2

\subsubsection{Properties of $\operatorname{Opt}_{0}\left(l, c_{m}\right)$}

For $\operatorname{Opt}_{0}\left(l, c_{m}\right)$, along with new results, we report existing results from Griewank and Walther [4] that we are using in this work. Note that most results from Griewank and Walther are adapted to the context here: in their work they only considered the number of re-execution of forward steps, while here we count the total execution time. In general this simply adds a cost of $l u_{f}+(l+1) u_{b}$ to the execution time.

Definition $6(\beta)$ We call $\beta$ the function

$$
\beta: x, y \mapsto\left(\begin{array}{c}
x+y \\
x
\end{array}\right)
$$

Note that $\beta$ is a critical parameter for all results from Griewank and Walther [4]. 
Lemma 2 (Griewank and Walther [4]) Let $c_{m} \in \mathbb{N}$, then $x \in \mathbb{N} \mapsto O p t_{0}\left(x, c_{m}\right)$ is convex. Theorem 3 (Griewank and Walther [4] (Proposition 1, Equation 3)) Let $l \in \mathbb{N}$ and $c_{m} \in \mathbb{N}$. The explicit form for $\mathrm{Opt}_{0}\left(l, c_{m}\right)$ is:

$$
O p t_{0}\left(l, c_{m}\right)=l \cdot(t+1) u_{f}-\beta\left(c_{m}+1, t-1\right) u_{f}+(l+1) u_{b},
$$

where $t$ is the unique integer satisfying $\beta\left(c_{m}, t-1\right) \leq l<\beta\left(c_{m}, t\right)$.

Theorem 4 (Griewank and Walther [4] (Proposition 1, Equation 4)) Let $l \in \mathbb{N}$ and $c_{m} \in \mathbb{N}$. Let $t$ be the unique integer satisfying $\beta\left(c_{m}, t-1\right) \leq l<\beta\left(c_{m}, t\right)$. Denote $j$ such that

$$
O p t_{0}\left(l, c_{m}\right)=j u_{f}+O p t_{0}\left(l-j, c_{m}-1\right)+O p t_{0}\left(j-1, c_{m}\right),
$$

then

$$
\beta\left(c_{m}, t-2\right) \leq j \leq \beta\left(c_{m}, t-1\right) .
$$

Lemma 3 Let $l \in \mathbb{N}, c_{m} \in \mathbb{N}$ and $t$ the unique integer satisfying $\beta\left(c_{m}, t-1\right)<l \leq$ $\beta\left(c_{m}, t\right)$. Then:

$$
\begin{array}{ll}
O p t_{0}\left(l+1, c_{m}\right)-O p t_{0}\left(l, c_{m}\right)=(t+1) \cdot u_{f}+u_{b} & \left(\text { if } l<\beta\left(c_{m}, t\right)\right) \\
O p t_{0}\left(l+1, c_{m}\right)-O p t_{0}\left(l, c_{m}\right)=(t+2) \cdot u_{f}+u_{b} & \left(\text { if } l=\beta\left(c_{m}, t\right)\right)
\end{array}
$$

Proof. Let $l \in \mathbb{N}, c_{m} \in \mathbb{N}$ and $t$ the unique integer satisfying $\beta\left(c_{m}, t-1\right)<l \leq \beta\left(c_{m}, t\right)$.

- If $l<\beta\left(c_{m}, t\right)$, then $t$ is also the unique integer satisfying $\beta\left(c_{m}, t-1\right)<l+1 \leq$ $\beta\left(c_{m}, t\right)$. The explicit form for $\operatorname{Opt}_{0}\left(l+1, c_{m}\right)$ (see Theorem 3$)$ is:

$$
\operatorname{Opt}_{0}\left(l+1, c_{m}\right)=(l+1) \cdot(t+1) \cdot u_{f}-\beta\left(c_{m}+1, t-1\right) \cdot u_{f}+(l+2) \cdot u_{b}
$$

Thus:

$$
\mathrm{Opt}_{0}\left(l+1, c_{m}\right)-\mathrm{Opt}_{0}\left(l, c_{m}\right)=(t+1) \cdot u_{f}+u_{b}
$$

- If $l=\beta\left(c_{m}, t\right)$, then $t$ satisfies $\beta\left(c_{m}, t\right)<l+1 \leq \beta\left(c_{m}, t+1\right)$. The explicit form for $\operatorname{Opt}_{0}\left(l+1, c_{m}\right)$ (see Theorem 3) is:

$$
\operatorname{Opt}_{0}\left(l+1, c_{m}\right)=(l+1) \cdot(t+2) \cdot u_{f}-\beta\left(c_{m}+1, t\right) \cdot u_{f}+(l+2) \cdot u_{b}
$$

Thus:

$$
\begin{aligned}
\operatorname{Opt}_{0}\left(l+1, c_{m}\right)-\mathrm{Opt}_{0}\left(l, c_{m}\right) & =\left(l+t+2+\beta\left(c_{m}+1, t-1\right)-\beta\left(c_{m}+1, t\right)\right) \cdot u_{f}+u_{b} \\
& =\left(l+t+2-\beta\left(c_{m}, t\right)\right) \cdot u_{f}+u_{b} \\
& =(t+2) \cdot u_{f}+u_{b}
\end{aligned}
$$


Corollary 2 Let $c_{m} \in \mathbb{N}$ and $t \in \mathbb{N}$.

Then for all $l \geq \beta\left(c_{m}, t\right)$ :

$$
O p t_{0}\left(l+1, c_{m}\right)-O p t_{0}\left(l, c_{m}\right) \geq(t+2) \cdot u_{f}+u_{b}
$$

Also, for all $l<\beta\left(c_{m}, t\right)$ :

$$
O p t_{0}\left(l+1, c_{m}\right)-O p t_{0}\left(l, c_{m}\right) \leq(t+1) \cdot u_{f}+u_{b}
$$

Proof. Obviously $t$ is the unique integer satisfying $\beta\left(c_{m}, t-1\right)<\beta\left(c_{m}, t\right) \leq \beta\left(c_{m}, t\right)$. Thus for all $t \in \mathbb{N}$ :

$$
\mathrm{Opt}_{0}\left(\beta\left(c_{m}, t\right)+1, c_{m}\right)-\mathrm{Opt}_{0}\left(\beta\left(c_{m}, t\right), c_{m}\right)=(t+2) \cdot u_{f}+u_{b}
$$

- Let $l \geq \beta\left(c_{m}, t\right)$. By convexity of function $x \mapsto \operatorname{Opt}_{0}\left(x, c_{m}\right)$ (Lemma 2):

$$
\begin{aligned}
\mathrm{Opt}_{0}\left(l+1, c_{m}\right)-\mathrm{Opt}_{0}\left(l, c_{m}\right) & \geq \mathrm{Opt}_{0}\left(\beta\left(c_{m}, t\right)+1, c_{m}\right)-\mathrm{Opt}_{0}\left(\beta\left(c_{m}, t\right), c_{m}\right) \\
& \geq(t+2) \cdot u_{f}+u_{b}
\end{aligned}
$$

- Let $l<\beta\left(c_{m}, t\right)$. If $l>\beta\left(c_{m}, t-1\right)$ then $t$ is the unique integer satisfying $\beta\left(c_{m}, t-\right.$ 1) $<l \leq \beta\left(c_{m}, t\right)$ and:

$$
\operatorname{Opt}_{0}\left(l+1, c_{m}\right)-\operatorname{Opt}_{0}\left(l, c_{m}\right)=(t+1) \cdot u_{f}+u_{b}
$$

If $l \leq \beta\left(c_{m}, t-1\right)$, By convexity of function $x \mapsto \operatorname{Opt}_{0}\left(x, c_{m}\right)$ :

$$
\begin{aligned}
\mathrm{Opt}_{0}\left(l+1, c_{m}\right)-\mathrm{Opt}_{0}\left(l, c_{m}\right) & \leq \mathrm{Opt}_{0}\left(\beta\left(c_{m}, t-1\right)+1, c_{m}\right)-\mathrm{Opt}_{0}\left(\beta\left(c_{m}, t-1\right), c_{m}\right) \\
& =(t+1) \cdot u_{f}+u_{b}
\end{aligned}
$$

\subsubsection{Properties of $O p t_{1}^{(d)}\left(l, c_{m}, r_{d}\right)$}

Contrarily to $\operatorname{Opt}_{0}\left(l, c_{m}\right)$, $\operatorname{Opt}_{1}^{(d)}\left(l, c_{m}, r_{d}\right)$ is not convex. This is mainly due to the fact that depending on the length of a period the number of disk reads can be different. However, we can extract some convexity from generalized functions where the number of disk reads is constant. This is the idea of the function $g_{n}$ presented in Definition 7 which is the execution time of a period if we were to do exactly $n$ disk reads.

Definition 7 In the following, for a fixed $c_{m} \in \mathbb{N}, w_{d} \in \mathbb{R}$ and $r_{d} \in \mathbb{R}$, let us denote:

$$
\begin{aligned}
g_{0}(l) & =\operatorname{Opt}_{0}\left(l, c_{m}\right) \\
\forall n>0, \quad & g_{n}(l)=\min _{0<j<l}\left\{g_{n-1}(j-1)+j u_{f}+\operatorname{Opt}_{0}\left(l-j, c_{m}\right)+r_{d}\right\}
\end{aligned}
$$

Note that for $n>0$ and $l=1$, we consider the common convention that the minimum over an empty set is $+\infty$. We can show formally that $\operatorname{Opt}_{1}^{(d)}\left(l, c_{m}, r_{d}\right)$ is the optimum execution time among the feasible solutions that do exactly $n \geq 0$ disk reads. 
LEMMA 4 For all $l$,

$$
O p t_{1}^{(d)}\left(l, c_{m}, r_{d}\right)=\min _{n \geq 0} g_{n}(l)
$$

Proof. We show the result by induction on $l$. The result is true for $l=1$, because $\mathrm{Opt}_{1}^{(d)}\left(1, c_{m}, r_{d}\right)=\operatorname{Opt}_{0}\left(1, c_{m}\right)=g_{0}(1)$ and for all $n>0, g_{n}(1)=+\infty$. Assume that for all $j<l, g(j)=\min _{n>0} g_{n}(j)$ and let us prove the result for $l$. By definition of $\mathrm{Opt}_{1}^{(d)}\left(l, c_{m}, r_{d}\right)($ Theorem 1 ),

$$
\begin{aligned}
& \mathrm{Opt}_{1}^{(d)}\left(l, c_{m}, r_{d}\right)=\min _{0<j<l}\left\{\begin{array}{l}
\mathrm{Opt}_{0}\left(l, c_{m}\right) \\
j u_{f}+\mathrm{Opt}_{0}\left(l-j, c_{m}\right)+r_{d}+\mathrm{Opt}_{1}^{(d)}\left(j-1, c_{m}, r_{d}\right)
\end{array}\right. \\
& =\min _{0<j<l}\left\{\begin{array}{l}
g_{0}(l) \\
j u_{f}+\mathrm{Opt}_{0}\left(l-j, c_{m}\right)+r_{d}+\min _{n \geq 0} g_{n}(j-1)
\end{array}\right. \\
& =\min _{n \geq 0}\left\{\begin{array}{l}
g_{0}(l) \\
\min _{0<j<l}\left\{j u_{f}+\mathrm{Opt}_{0}\left(l-j, c_{m}\right)+r_{d}+g_{n}(j-1)\right\}
\end{array}\right. \\
& =\min _{n \geq 0}\left(g_{0}(l), g_{n+1}(l)\right) \\
& =\min _{n \geq 0} g_{n}(l)
\end{aligned}
$$

Lemma 5 Let $c_{m} \in \mathbb{N}$ and $r_{d} \in \mathbb{R}$. For all $l \in \mathbb{N}$ and $n>0$, the function

$$
h_{n, l}: j \mapsto j u_{f}+O p t_{0}\left(l-j, c_{m}\right)+r_{d}+g_{n-1}(j-1)
$$

is convex.

Proof. We first show that $g_{n}$ is convex. Clearly $g_{0}$ is convex (Lemma 2). We then show the result by induction on $n$. Assume for $n>0$ that $g_{n-1}$ is convex. By definition, $g_{n}$ is the infimal convolution ${ }^{2}$ of function $x \mapsto g_{n-1}(x)+(x+1) u_{f}+r_{d}$ and $l \mapsto \mathrm{Opt}_{0}\left(l, c_{m}\right)$ which are both convex. Hence for all $n, g_{n}$ is convex [6]. So is $h_{n, l}$ as sum of convex functions.

Finally, we can give an estimate of the number of forward steps done by 1D-REVOLVE when it does not behave as REVOLVE:

Lemma 6 Given $l$, such that $O p t_{1}^{(d)}\left(l, c_{m}, r_{d}\right)<O p t_{0}\left(l, c_{m}\right)$. Consider $n^{\star}>0$ and the largest $j^{\star}$ such that

$$
O p t_{1}^{(d)}\left(l, c_{m}, r_{d}\right)=h_{n^{\star}, l}\left(j^{\star}\right)
$$

where $h_{n^{\star}, l}$ is defined by Equation (9). Then:

$$
j^{\star} \geq \beta\left(c_{m}, t-1\right)
$$

\footnotetext{
${ }^{2}$ The infimal convolution of two functions $f$ and $g$ is defined as $f \square g(l)=\min \{f(l-x)+g(x) \mid x \in\{0, \ldots l\}\}$
} 
where $t$ is the unique integer satisfying $\beta\left(c_{m}, t-1\right)<\frac{l}{2} \leq \beta\left(c_{m}, t\right)$.

Proof. Since function $h_{n^{\star}, l}$ is convex (Lemma 5), its minimum is reached on a segment. Thus, we just have to prove that $h_{n^{\star}, l}\left(\beta\left(c_{m}, t-1\right)-1\right) \geq h_{n^{\star}, l}\left(\beta\left(c_{m}, t-1\right)\right)$ to prove that the largest element of this segment (namely $\left.j^{\star}\right)$ is larger than $\beta\left(c_{m}, t-1\right)$. For simplicity let us note $y=\beta\left(c_{m}, t-1\right)$.

Assume first that $n^{\star}=1$.

$$
\begin{aligned}
& h_{1, l}(y-1)-h_{1, l}(y)= \\
& \quad-u_{f}+\operatorname{Opt}_{0}\left(l-y+1, c_{m}\right)-\mathrm{Opt}_{0}\left(l-y, c_{m}\right)+\mathrm{Opt}_{0}\left(y-2, c_{m}\right)-\mathrm{Opt}_{0}\left(y-1, c_{m}\right)
\end{aligned}
$$

Because $y<\frac{l}{2}$, then $l-y>\frac{l}{2}>\beta\left(c_{m}, t-1\right)$. By Corollary 2

$$
\mathrm{Opt}_{0}\left(l-y+1, c_{m}\right)-\mathrm{Opt}_{0}\left(l-y, c_{m}\right) \geq(t+1) u_{f}+u_{b} .
$$

Besides since $y=\beta\left(c_{m}, t-1\right)$, then $y-2<\beta\left(c_{m}, t-1\right)$. By Corollary 2

$$
\mathrm{Opt}_{0}\left(y-1, c_{m}\right)-\mathrm{Opt}_{0}\left(y-2, c_{m}\right) \leq t \cdot u_{f}+u_{b} \text {. }
$$

Finally, we get:

$$
\begin{aligned}
h_{1, l}(y-1)-h_{1, l}(y) & \geq-u_{f}+(t+1) u_{f}+u_{b}-\left(t \cdot u_{f}+u_{b}\right) \\
& \geq 0
\end{aligned}
$$

which proves that $j^{\star} \geq \beta\left(c_{m}, t-1\right)$.

Let us now assume that $n^{\star} \geq 2$.

$$
\begin{aligned}
& h_{n^{\star}, l}(y-1)-h_{n^{\star}, l}(y)= \\
& \quad-u_{f}+\mathrm{Opt}_{0}\left(l-y+1, c_{m}\right)-\mathrm{Opt}_{0}\left(l-y, c_{m}\right)+g_{n-1}(y-2)-g_{n-1}(y-1)
\end{aligned}
$$

Let $j \in\{1, \ldots, y-3\}$ such that:

$$
g_{n-1}(y-2)=g_{n-2}(j-1)+j u_{f}+\operatorname{Opt}_{0}\left(y-2-j, c_{m}\right)+r_{d}
$$

Clearly, since $j \in\{1, \ldots, y-2\}$ :

$$
g_{n-1}(y-1) \leq g_{n-2}(j-1)+j u_{f}+\operatorname{Opt}_{0}\left(y-1-j, c_{m}\right)+r_{d}
$$

Thus:

$$
\begin{aligned}
& h_{n^{\star}, l}(y-1)-h_{n^{\star}, l}(y)= \\
& \quad-u_{f}+\mathrm{Opt}_{0}\left(l-y+1, c_{m}\right)-\mathrm{Opt}_{0}\left(l-y, c_{m}\right)+\mathrm{Opt}_{0}\left(y-2-j, c_{m}\right)-\mathrm{Opt}_{0}\left(y-1-j, c_{m}\right)
\end{aligned}
$$


Because $y<\frac{l}{2}$, then $l-y>\frac{l}{2}>\beta\left(c_{m}, t-1\right)$. By Corollary 2 ;

$$
\mathrm{Opt}_{0}\left(l-y+1, c_{m}\right)-\operatorname{Opt}_{0}\left(l-y, c_{m}\right) \geq(t+1) u_{f}+u_{b} .
$$

Besides since $y=\beta\left(c_{m}, t-1\right)$, then $y-2-j<\beta\left(c_{m}, t-1\right)$. By Corollary 2

$$
\operatorname{Opt}_{0}\left(y-1-j, c_{m}\right)-\operatorname{Opt}_{0}\left(y-2-j, c_{m}\right) \leq t \cdot u_{f}+u_{b} .
$$

Finally, we get:

$$
\begin{aligned}
h_{n^{\star}, l}(y-1)-h_{n^{\star}, l}(y) & \geq-u_{f}+(t+1) u_{f}+u_{b}-\left(t \cdot u_{f}+u_{b}\right) \\
& \geq 0,
\end{aligned}
$$

which proves that $j^{\star} \geq \beta\left(c_{m}, t-1\right)$.

Finally, we present another useful result: if 1D-REvolve behave as REvolve for a given $l$, then it will also do the same for all $l^{\prime}<l$ :

LEMMA 7 For all $l$, if $O p t_{1}^{(d)}\left(l, c_{m}, r_{d}\right)=O p t_{0}\left(l, c_{m}\right)$, then for all $l^{\prime}<l$, $O p t_{1}^{(d)}\left(l^{\prime}, c_{m}, r_{d}\right)=O p t_{0}\left(l^{\prime}, c_{m}\right)$.

Proof. We show the result by contradiction. Assume there exists $l^{\prime}<l$ such that $\mathrm{Opt}_{1}^{(d)}\left(l^{\prime}, c_{m}, r_{d}\right)<\operatorname{Opt}_{0}\left(l^{\prime}, c_{m}\right)$. Then by Theorem 1, there exists $j<l^{\prime}$ and

$$
\mathrm{Opt}_{1}^{(d)}\left(l^{\prime}, c_{m}, r_{d}\right)=j u_{f}+\mathrm{Opt}_{0}\left(l^{\prime}-j, c_{m}\right)+r_{d}+\mathrm{Opt}_{1}^{(d)}\left(j-1, c_{m}, r_{d}\right)<\mathrm{Opt}_{0}\left(l^{\prime}, c_{m}\right)
$$

Because $\operatorname{Opt}_{1}^{(d)}\left(l, c_{m}, r_{d}\right)=\operatorname{Opt}_{0}\left(l, c_{m}\right)$, then by Theorem 1

$$
\mathrm{Opt}_{0}\left(l, c_{m}\right) \leq j u_{f}+\mathrm{Opt}_{0}\left(l-j, c_{m}\right)+r_{d}+\mathrm{Opt}_{1}^{(d)}\left(j-1, c_{m}, r_{d}\right) .
$$

Finally, combining Eq. (10) and (11) we get:

$$
\mathrm{Opt}_{0}\left(l, c_{m}\right)-\mathrm{Opt}_{0}\left(l^{\prime}, c_{m}\right)<\operatorname{Opt}_{0}\left(l-j, c_{m}\right)-\mathrm{Opt}_{0}\left(l^{\prime}-j, c_{m}\right)
$$

which is absurd by convexity of $l \mapsto \operatorname{Opt}_{0}\left(l, c_{m}\right)$ (Lemma 2).

\section{$4.2 \quad$ Execution time of a $D S$}

In this section, we provide several basic results on the execution times of the Dominant Sequences that will be useful in the rest of the paper. We remind that given $\mathcal{S}=\left(m_{1}, \cdots, m_{n_{l}} ;\right.$ res $)$ a DS, we denote by $\mathcal{E} x e c(\mathcal{S})$ or $\mathcal{E} x e c\left(m_{1}, \cdots, m_{n_{l}} ;\right.$ res $)$ its execution time.

Theorem 5 Let $\mathcal{S}=\left(m_{1}, \cdots, m_{n_{l}} ;\right.$ res $)$ a DS and $l=\sum_{i=1}^{n_{l}} m_{i}+$ res. Its execution time is:

$$
\mathcal{E} x e c(\mathcal{S})=(l-r e s) u_{f}+O p t_{0}\left(r e s, c_{m}\right)+\sum_{i=1}^{n_{l}}\left(w_{d}+r_{d}+O p t_{1}^{(d)}\left(m_{i}-1, c_{m}, r_{d}\right)\right)
$$


Proof. By definition of Algorithm 2, the execution time of $\mathcal{S}$ is:

$$
\begin{aligned}
\mathcal{E} x e c(\mathcal{S}) & =\sum_{i=1}^{n_{l}}\left(w_{d}+m_{i} u_{f}\right)+\mathrm{Opt}_{0}\left(\mathrm{res}, c_{m}\right)+\sum_{i=1}^{n_{l}}\left(r_{d}+\mathrm{Opt}_{1}^{(d)}\left(m_{i}-1, c_{m}, r_{d}\right)\right) \\
& =(l-\mathrm{res}) u_{f}+\mathrm{Opt}_{0}\left(\mathrm{res}, c_{m}\right)+\sum_{i=1}^{n_{l}}\left(r_{d}+w_{d}+\mathrm{Opt}_{1}^{(d)}\left(m_{i}-1, c_{m}, r_{d}\right)\right)
\end{aligned}
$$

Corollary 3 Given $n_{l} \geq 1$ and $\mathcal{S}=\left(m_{1}, \cdots, m_{n_{l}} ;\right.$ res $)$ a DS. Then:

$$
\mathcal{E} x e c(\mathcal{S})=m_{1} u_{f}+w_{d}+r_{d}+O p t_{1}^{(d)}\left(m_{1}-1, c_{m}, r_{d}\right)+\mathcal{E} x e c\left(m_{2}, \cdots, m_{n_{l}} ; \text { res }\right)
$$

Proof. Note first that by definition, $\left(m_{2}, \cdots, m_{n_{l}} ;\right.$ res $)$ is a DS for $\operatorname{PROB}_{\infty}(l-$ $\left.m_{1}, c_{m}, w_{d}, r_{d}\right)$. Then we have:

$$
\begin{aligned}
\mathcal{E x e c}(\mathcal{S})= & (l-\mathrm{res}) u_{f}+\mathrm{Opt}_{0}\left(\mathrm{res}, c_{m}\right)+\sum_{i=1}^{n_{l}}\left(w_{d}+r_{d}+\mathrm{Opt}_{1}^{(d)}\left(m_{i}-1, c_{m}, r_{d}\right)\right) \\
= & \left(l-m_{1}-\mathrm{res}\right) u_{f}+m_{1} u_{f}+\mathrm{Opt}_{0}\left(\mathrm{res}, c_{m}\right)+\sum_{i=1}^{n_{l}}\left(w_{d}+r_{d}+\mathrm{Opt}_{1}^{(d)}\left(m_{i}-1, c_{m}, r_{d}\right)\right) \\
= & m_{1} u_{f}+\left(w_{d}+r_{d}+\mathrm{Opt}_{1}^{(d)}\left(m_{1}-1, c_{m}, r_{d}\right)\right)+\left(l-m_{1}-\mathrm{res}\right) u_{f}+\mathrm{Opt}_{0}\left(\mathrm{res}, c_{m}\right) \\
& \quad+\sum_{i=2}^{n_{l}}\left(w_{d}+r_{d}+\mathrm{Opt}_{1}^{(d)}\left(m_{i}-1, c_{m}, r_{d}\right)\right) \\
= & m_{1} u_{f}+w_{d}+r_{d}+\mathrm{Opt}_{1}^{(d)}\left(m_{1}-1, c_{m}, r_{d}\right)+\mathcal{E x e c}\left(m_{2}, \cdots, m_{n_{l}} ; \mathrm{res}\right)
\end{aligned}
$$

Corollary 4 Let $l \in \mathbb{N}, \mathcal{S}=\left(m_{1}, \cdots, m_{n_{l}} ;\right.$ res $)$ a $D S$ for $\operatorname{PROB}_{\infty}\left(l, c_{m}, w_{d}, r_{d}\right)$, and $\mathcal{S}_{+1}=\left(m_{1}, \cdots, m_{n_{l}} ;\right.$ res +1$)$, then

$$
\mathcal{E} x e c\left(\mathcal{S}_{+1}\right)-\mathcal{E} x e c(\mathcal{S})=O p t_{0}\left(r e s+1, c_{m}\right)-O p t_{0}\left(r e s, c_{m}\right)
$$

Proof. This is a direct consequence of Equation 12):

$$
\begin{aligned}
\mathcal{E x e c}(\mathcal{S}) & =(l-\mathrm{res}) u_{f}+\mathrm{Opt}_{0}\left(\mathrm{res}, c_{m}\right)+\sum_{i=1}^{n_{l}}\left(w_{d}+r_{d}+\mathrm{Opt}_{1}^{(d)}\left(m_{i}-1, c_{m}, r_{d}\right)\right) \\
\mathcal{E} x e c\left(\mathcal{S}_{+1}\right) & =((l+1)-(\operatorname{res}+1)) u_{f}+\mathrm{Opt}_{0}\left(\mathrm{res}+1, c_{m}\right)+\sum_{i=1}^{n_{l}}\left(w_{d}+r_{d}+\mathrm{Opt}_{1}^{(d)}\left(m_{i}-1, c_{m}, r_{d}\right)\right)
\end{aligned}
$$




\subsection{Existence of a DOS}

In this section, we prove that for every values $l, c_{m}, r_{d}$ and $w_{d}$ there is a Dominant Sequence returned by ALGoDom that is optimal for $\operatorname{PROB}_{\infty}\left(l, c_{m}, w_{d}, r_{d}\right)$. These sequences are called Dominant Optimal Sequences (DOS).

THEOREM 6 Given an adjoint computation chain of size $l \in \mathbb{N}, c_{m} \in \mathbb{N}$ memory slots, a cost $w_{d} \geq 0$ to write to disk and a cost $r_{d} \geq 0$ to read from disk, then there exists $n_{l} \in \mathbb{N}$ and $\left(m_{1}, \cdots, m_{n_{l}}\right.$, res $) \in \mathbb{N}^{n_{l}+1}$, such that:

- $l=\sum_{i=1}^{n_{l}} m_{i}+$ res;

- Sequence $\operatorname{AlgoDom}\left(m_{1}, \cdots, m_{n_{l}} ;\right.$ res $)$ is optimal for $\operatorname{PROB}_{\infty}\left(l, c_{m}, w_{d}, r_{d}\right)$.

Proof. We now show the existence of $n_{l} \in \mathbb{N}$ and $\left(m_{1}, \cdots, m_{n_{l}}\right.$, res $) \in \mathbb{N}^{n_{l}+1}$ such that $l=\sum_{i=1}^{n_{l}} m_{i}+$ res and $\mathcal{E} x e c\left(m_{1}, \cdots, m_{n_{l}} ;\right.$ res $)=\operatorname{Opt}_{\infty}\left(l, c_{m}, w_{d}, r_{d}\right)$.

First let us remind the optimal execution time for $\operatorname{PROB}_{\infty}\left(l, c_{m}, w_{d}, r_{d}\right)$ (Theorem 1):

$$
\begin{aligned}
& \mathrm{Opt}_{\infty}\left(l, c_{m}, w_{d}, r_{d}\right)= \\
& \min \left\{\begin{array}{l}
\mathrm{Opt}_{0}\left(l, c_{m}\right) \\
w_{d}+\min _{1 \leq j \leq l-1}\left\{j u_{f}+\mathrm{Opt}_{\infty}\left(l-j, c_{m}, w_{d}, r_{d}\right)+r_{d}+\mathrm{Opt}_{1}^{(d)}\left(j-1, c_{m}, r_{d}\right)\right\}
\end{array}\right. \\
& \mathrm{Opt}_{1}^{(d)}\left(l, c_{m}, r_{d}\right)= \\
& \min \left\{\begin{array}{l}
\mathrm{Opt}_{0}\left(l, c_{m}\right) \\
\min _{1 \leq j \leq l-1}\left\{j u_{f}+\mathrm{Opt}_{0}\left(l-j, c_{m}\right)+r_{d}+\mathrm{Opt}_{1}^{(d)}\left(j-1, c_{m}, r_{d}\right)\right\}
\end{array}\right.
\end{aligned}
$$

We now show the main result by induction on $l$. For $l=1$, then $\operatorname{Opt}_{\infty}\left(l, c_{m}, w_{d}, r_{d}\right)=$ $\operatorname{Opt}_{0}\left(l, c_{m}\right)$ and $n_{l}=0$, and $(; l)$ is optimal for $\operatorname{PROB}_{\infty}\left(l, c_{m}, w_{d}, r_{d}\right)$. Assume the result is true for all $l^{\prime}<l$. Let us show the result for $l$.

- If $\operatorname{Opt}_{\infty}\left(l, c_{m}, w_{d}, r_{d}\right)=\operatorname{Opt}_{0}\left(l, c_{m}\right)$ then we choose $n_{l}=0$ and $(; l)$ is optimal for $\operatorname{PROB}_{\infty}\left(l, c_{m}, w_{d}, r_{d}\right)$.

- Otherwise, then there exists $m_{1}$, such that

$$
\operatorname{Opt}_{\infty}\left(l, c_{m}, w_{d}, r_{d}\right)=w_{d}+m_{1} u_{f}+\mathrm{Opt}_{\infty}\left(l-m_{1}, c_{m}, w_{d}, r_{d}\right)+r_{d}+\mathrm{Opt}_{1}^{(d)}\left(m_{1}-1, c_{m}, r_{d}\right) .
$$

By induction hypothesis, there exists $\left(m_{2}, \cdots, m_{n_{l-m_{1}}+1}\right.$, res $)$ such that

○ $\sum_{i=2}^{n_{l-m_{1}}+1} m_{i}+$ res $=l-m_{1}$

○ $\left(m_{2}, \cdots, m_{n_{l-m_{1}+1}}\right.$; res $)$ is optimal for $\operatorname{PROB}_{\infty}\left(l-m_{1}, c_{m}, w_{d}, r_{d}\right)$.

In particular, we have:

$$
\begin{aligned}
\operatorname{Opt}_{\infty}\left(l, c_{m}, w_{d}, r_{d}\right) & =w_{d}+m_{1} u_{f}+\mathrm{Opt}_{\infty}\left(l-m_{1}, c_{m}, w_{d}, r_{d}\right)+r_{d}+\mathrm{Opt}_{1}^{(d)}\left(m_{1}-1, c_{m}, r_{d}\right) \\
& =w_{d}+m_{1} u_{f}+\mathcal{E} x e c\left(m_{2}, \cdots, m_{n_{l-m_{1}}+1} ; \mathrm{res}\right)+r_{d}+\mathrm{Opt}_{1}^{(d)}\left(m_{1}-1, c_{m}, r_{d}\right) \\
& =\mathcal{E} x e c\left(m_{1}, \cdots, m_{n_{l}} ; \text { res }\right)
\end{aligned}
$$

The last equality is a consequence of Corollary 3. Finally, this shows that $n_{l},\left(m_{1}, m_{2}, \cdots, m_{n_{l-m_{1}}+1}\right.$, res $)$ are such that $\left(m_{1}, \cdots, m_{n_{l}}\right.$;res $)$ is optimal for $\operatorname{PROB}_{\infty}\left(l, c_{m}, w_{d}, r_{d}\right)$, hence showing the result. 
Corollary 5 Let $n_{l} \geq 1$ and $\mathcal{S}=\left(m_{1}, \cdots, m_{n_{l}} ;\right.$ res $)$ a DOS for $\mathrm{PROB}_{\infty}\left(l, c_{m}, w_{d}, r_{d}\right)$ with $l=\sum_{i=1}^{n_{l}} m_{i}+$ res. Then:

(1) For all permutation $\sigma \in S_{n_{l}}$, the dominant sequence $\mathcal{S}_{\sigma}=\left(m_{\sigma(1)}, \cdots, m_{\sigma\left(n_{l}\right)}\right.$; res) is a DOS for $\operatorname{PROB}_{\infty}\left(l, c_{m}, w_{d}, r_{d}\right)$.

(2) The dominant sequence $\mathcal{S}_{-m_{1}}=\left(m_{2}, \cdots, m_{n_{l}}\right.$;res $)$ is a DOS for $\mathrm{PROB}_{\infty}(l-$ $\left.m_{1}, c_{m}, w_{d}, r_{d}\right)$.

(3) Given $n_{l^{\prime}}$ and $\mathcal{S}^{\prime}=\left(m_{1}^{\prime}, \cdots, m_{n_{l^{\prime}}}^{\prime}\right.$;res' $)$ a DOS for $\operatorname{PROB}_{\infty}\left(l-m_{1}, c_{m}, w_{d}, r_{d}\right)$, then $\tilde{\mathcal{S}}=\left(m_{1}, m_{1}^{\prime}, \cdots, m_{n_{l^{\prime}}}^{\prime} ;\right.$ res $\left.s^{\prime}\right)$ is a DOS for $\operatorname{PROB}_{\infty}\left(l, c_{m}, w_{d}, r_{d}\right)$.

Proof. Based on Equation (12), $\mathcal{S}_{\sigma}$ and $\mathcal{S}$ have the same execution time. Thus, if $\mathcal{S}$ is an optimal solution to $\operatorname{PROB}_{\infty}\left(l, c_{m}, w_{d}, r_{d}\right)$, so is $\mathcal{S}_{\sigma}$.

We prove the two next points together. First, by definition $\mathcal{S}_{-m_{1}}$ is a DS for $\operatorname{PROB}_{\infty}(l-$ $\left.m_{1}, c_{m}, w_{d}, r_{d}\right)$. Because $\mathcal{S}^{\prime}$ is a DOS for $\operatorname{PROB}_{\infty}\left(l-m_{1}, c_{m}, w_{d}, r_{d}\right)$, then by optimality,

$$
\mathcal{E} x e c\left(\mathcal{S}^{\prime}\right)-\mathcal{E} x e c\left(\mathcal{S}_{-m_{1}}\right) \leq 0 .
$$

Furthermore, by definition, $\tilde{\mathcal{S}}$ is a DS for $\operatorname{PROB}_{\infty}\left(l, c_{m}, w_{d}, r_{d}\right)$, hence

$$
\mathcal{E} x e c(\mathcal{S})-\mathcal{E} x e c(\tilde{\mathcal{S}}) \leq 0 .
$$

According to Corollary 3 ,

$$
\begin{aligned}
& \mathcal{E} x e c(\tilde{\mathcal{S}})=m_{1} u_{f}+w_{d}+r_{d}+\mathrm{Opt}_{1}^{(d)}\left(m_{1}-1, c_{m}, r_{d}\right)+\mathcal{E x e c}\left(\mathcal{S}^{\prime}\right) \\
& \mathcal{E} x e c(\mathcal{S})=m_{1} u_{f}+w_{d}+r_{d}+\operatorname{Opt}_{1}^{(d)}\left(m_{1}-1, c_{m}, r_{d}\right)+\mathcal{E} x e c\left(\mathcal{S}_{-m_{1}}\right)
\end{aligned}
$$

Hence, $0 \leq \mathcal{E} x e c(\tilde{\mathcal{S}})-\mathcal{E} x e c(\mathcal{S})=\mathcal{E} x e c\left(\mathcal{S}^{\prime}\right)-\mathcal{E} x e c\left(\mathcal{S}_{-m_{1}}\right) \leq 0$, and finally we obtain $\mathcal{E} x e c(\tilde{\mathcal{S}})=\mathcal{E} x e c(\mathcal{S})$ and $\mathcal{E} x e c\left(\mathcal{S}^{\prime}\right)=\mathcal{E} x e c\left(\mathcal{S}_{-m_{1}}\right)$. Hence, $\tilde{\mathcal{S}}$ is a DOS for $\operatorname{PROB}_{\infty}\left(l, c_{m}, w_{d}, r_{d}\right)$, and $\mathcal{S}_{-m_{1}}$ is a DOS for $\operatorname{PROB}_{\infty}\left(l-m_{1}, c_{m}, w_{d}, r_{d}\right)$.

\subsection{Admissible periods $\mathcal{M}_{X}^{(A d m)}$}

Definition 8 (Admissible periods, $\mathcal{M}_{X}^{(\mathrm{Adm})}$ ) We call the set of admissible periods $\mathcal{M}_{X}^{(\mathrm{Adm})}$ :

$\mathcal{M}_{X}^{(\mathrm{Adm})}=\left\{m \in \mathbb{N} \mid\right.$ there exists $l$ and $\mathcal{S}$ a DOS for $\operatorname{PROB}_{\infty}\left(l, c_{m}, w_{d}, r_{d}\right)$ s.t. $m$ is a period of $\left.\mathcal{S}\right\}$

In this section, we want to show properties of the set of admissible periods, in particular our goal is to show that it is bounded independently of the size of the adjoint computation.

Definition 9 (Decomposition) We say that $m$ can be decomposed into $\left[\left[m_{1}: m_{2}\right]\right]$ if, $m=m_{1}+m_{2}$ and

$$
\mathrm{Opt}_{1}^{(d)}\left(m-1, c_{m}, r_{d}\right)>\mathrm{Opt}_{1}^{(d)}\left(m_{1}-1, c_{m}, r_{d}\right)+w_{d}+r_{d}+\mathrm{Opt}_{1}^{(d)}\left(m_{2}-1, c_{m}, r_{d}\right)
$$

Intuitively, this means that there will be no DOS with a period of size $m$, two periods of size $m_{1}, m_{2}$ will be preferred. Let us write formally this result. 
Theorem 7 (Decomposition) If $m$ can be decomposed into $\left[\left[m_{1}: m_{2}\right]\right]$, then there are no DOS with a period of size $m$ (i.e., $m \notin \mathcal{M}_{X}^{(A d m)}$ ).

Proof. We show the result by contradiction. Let $m$ be a period that can be decomposed into $\left[\left[m_{1}: m_{2}\right]\right]$. Assume there exists $l$ that admits a DOS with a period equal to $m$. Without loss of generality, let $\mathcal{S}=\left(m, m_{3}, \cdots, m_{n_{l}}\right.$; res $)$ denote this DOS.

Clearly, $\tilde{\mathcal{S}}=\left(m_{1}, \cdots, m_{n_{l}}\right.$;res $)$ is a DS for $\operatorname{PROB}_{\infty}\left(l, c_{m}, w_{d}, r_{d}\right)$. Let us show that $\mathcal{E} x e c(\mathcal{S})>\mathcal{E} x e c(\tilde{\mathcal{S}})$ which would contradict the optimality of $\mathcal{S}$.

$$
\begin{aligned}
& \mathcal{E} x e c(\tilde{\mathcal{S}})=(l-\operatorname{res}) u_{f}+\mathrm{Opt}_{0}\left(\mathrm{res}, c_{m}\right)+\sum_{i=1}^{n_{l}}\left(w_{d}+r_{d}+\mathrm{Opt}_{1}^{(d)}\left(m_{i}-1, c_{m}, r_{d}\right)\right) \\
& <(l-\mathrm{res}) u_{f}+\mathrm{Opt}_{0}\left(\mathrm{res}, c_{m}\right)+\sum_{i=3}^{n_{l}}\left(w_{d}+r_{d}+\mathrm{Opt}_{1}^{(d)}\left(m_{i}-1, c_{m}, r_{d}\right)\right) \\
& +\left(w_{d}+r_{d}+\mathrm{Opt}_{1}^{(d)}\left(m-1, c_{m}, r_{d}\right)\right) \\
& <\mathcal{E x e c}(\mathcal{S})
\end{aligned}
$$

Hence the result.

This decomposition allows us to characterize periods that are not admissible.

Corollary 6 Given $m \in \mathcal{M}_{X}^{(\text {Adm) }}$. If

$O p t_{1}^{(d)}\left(m-1, c_{m}, r_{d}\right)=j u_{f}+O p t_{0}\left(m-1-j, c_{m}\right)+O p t_{0}\left(j-1, c_{m}\right), \quad$ then $j \leq \frac{w_{d}+r_{d}}{u_{f}}$

$O p t_{1}^{(d)}\left(m-1, c_{m}, r_{d}\right)=j u_{f}+O p t_{0}\left(m-1-j, c_{m}\right)+r_{d}+O p t_{1}^{(d)}\left(j-1, c_{m}, r_{d}\right), \quad$ then $j \leq \frac{w_{d}}{u_{f}}$

Proof. We show the results by contradiction.

Let us consider Equation (16), i.e. $\mathrm{Opt}_{1}^{(d)}\left(m-1, c_{m}, r_{d}\right)=\mathrm{Opt}_{0}\left(m-1, c_{m}\right)$. Assume $j>\frac{w_{d}+r_{d}}{u_{f}}$. Because we have $\mathrm{Opt}_{1}^{(d)}\left(m-1, c_{m}, r_{d}\right)=\mathrm{Opt}_{0}\left(m-1, c_{m}\right)$, by Lemma 7 .

$$
\begin{aligned}
\mathrm{Opt}_{1}^{(d)}\left(m-1-j, c_{m}, r_{d}\right) & =\operatorname{Opt}_{0}\left(m-1-j, c_{m}\right) \quad \text { and } \\
\mathrm{Opt}_{1}^{(d)}\left(j-1, c_{m}, r_{d}\right) & =\operatorname{Opt}_{0}\left(j-1, c_{m}\right) .
\end{aligned}
$$

Consequently,

$$
\mathrm{Opt}_{1}^{(d)}\left(m-1, c_{m}, r_{d}\right)>w_{d}+r_{d}+\mathrm{Opt}_{1}^{(d)}\left(m-1-j, c_{m}, r_{d}\right)+\mathrm{Opt}_{1}^{(d)}\left(j-1, c_{m}, r_{d}\right)
$$

and $m$ can be decomposed into [[j:m-j]] and the result follows from Theorem 7 .

Let us now consider Equation (17), i.e. $\mathrm{Opt}_{1}^{(d)}\left(m-1, c_{m}, r_{d}\right)<\mathrm{Opt}_{0}\left(m-1, c_{m}\right)$. Assume $j>\frac{w_{d}}{u_{f}}$, then similarly it suffices to see that $m$ can be decomposed into $[[j: m-j]]$ (because $\left.\operatorname{Opt}_{0}\left(m-1-j, c_{m}\right) \geq \operatorname{Opt}_{1}^{(d)}\left(m-1-j, c_{m}, r_{d}\right)\right)$ and the result follows from Theorem 7. 
Corollary 7 Let $m \in \mathcal{M}_{X}^{(A d m)}$, then:

$$
O p t_{1}^{(d)}\left(m-1, c_{m}, r_{d}\right)=\min _{1 \leq j \leq \min \left(\frac{w_{d}}{u_{f}}, l-1\right)}\left\{\begin{array}{l}
O p t_{0}\left(m-1, c_{m}\right) \\
j u_{f}+O p t_{0}\left(m-1-j, c_{m}\right)+r_{d}+O p t_{1}^{(d)}\left(j-1, c_{m}, r_{d}\right)
\end{array}\right.
$$

Proof. This is a direct corollary from Corollary 6

Note that while Corollary 7 is not directly involved in the proof of Theorem 2, it provides a faster execution of $\mathrm{Opt}_{1}^{(d)}\left(l, c_{m}, r_{d}\right)$.

\subsection{Bounding the size of a period}

The main result of this section is that, for a given platform (defined by its parameters $c_{m}, w_{d}$, and $r_{d}$ ) the set of admissible periods is actually bounded. We prove this result by showing that admissible periods admit an upper bound on their size.

\subsubsection{Maximum period size}

Lemma 8 Let $t_{d}^{1}$ and $t_{d}^{2}$ be the only integers such that:

$$
\begin{aligned}
& \beta\left(c_{m}, t_{d}^{1}\right)>\frac{w_{d}+r_{d}}{u_{f}} \geq \beta\left(c_{m}, t_{d}^{1}-1\right), \\
& \beta\left(c_{m}, t_{d}^{2}\right) \geq \frac{w_{d}}{u_{f}}>\beta\left(c_{m}, t_{d}^{2}-1\right),
\end{aligned}
$$

then all admissible periods in $\mathcal{M}_{X}^{(\text {Adm) }}$ are smaller than

$$
m_{\max }=\max \left(\beta\left(c_{m}, t_{d}^{1}+1\right), 2 \beta\left(c_{m}, t_{d}^{2}\right)+1\right)
$$

Proof. Let $m \geq m_{\max }$.

Assume first that $\mathrm{Opt}_{1}^{(d)}\left(m-1, c_{m}, r_{d}\right)=\mathrm{Opt}_{0}\left(m-1, c_{m}\right)$. Then $\mathrm{Opt}_{1}^{(d)}\left(m-1, c_{m}, r_{d}\right)=$ $j_{m} u_{f}+\operatorname{Opt}_{0}\left(m-j_{m}-1, c_{m}\right)+\operatorname{Opt}_{0}\left(j_{m}-1, c_{m}\right)$, where $j_{m} \geq \beta\left(c_{m}, t_{d}^{1}\right)>\frac{w_{d}+r_{d}}{u_{f}}$ (Theorem 4). Then, by Corollary 6. $m \notin \mathcal{M}_{X}^{(\mathrm{Adm})}$.

Otherwise, assume $\operatorname{Opt}_{1}^{(d)}\left(m-1, c_{m}, r_{d}\right)<\operatorname{Opt}_{0}\left(m-1, c_{m}\right)$. According to Lemma 6 there exists $j_{m} \geq \beta\left(c_{m}, t_{d}^{2}\right) \geq \frac{w_{d}}{u_{f}}$ and $n_{m}$ such that:

$$
\mathrm{Opt}_{1}^{(d)}\left(m-1, c_{m}, r_{d}\right)=j_{m} u_{f}+\mathrm{Opt}_{0}\left(m-j_{m}-1, c_{m}\right)+r_{d}+g_{n_{m}-1}\left(j_{m}-1\right)
$$

In particular, by Definition 7, we know that $g_{n_{m}-1}\left(j_{m}-1\right)=\min _{n} g_{n}\left(j_{m}-1\right)$. And by Lemma 4. $g_{n_{m}-1}\left(j_{m}-1\right)=\mathrm{Opt}_{1}^{(d)}\left(j_{m}-1, c_{m}, r_{d}\right)$. Thus:

$$
\mathrm{Opt}_{1}^{(d)}\left(m-1, c_{m}, r_{d}\right)=j_{m} u_{f}+\mathrm{Opt}_{0}\left(m-j_{m}-1, c_{m}\right)+r_{d}+\mathrm{Opt}_{1}^{(d)}\left(j_{m}-1, c_{m}, r_{d}\right)
$$

Then, by Corollary 6, $m \notin \mathcal{M}_{X}^{(\mathrm{Adm})}$. 
TheOREM 8 The number of admissible periods $\left|\mathcal{M}_{X}^{(\text {Adm) }}\right|$ is bounded.

Proof. This theorem is a corollary of Lemma 8 there are at most $m_{\max }$ periods in $\mathcal{M}_{X}^{(\mathrm{Adm})}$ and $\mathcal{M}_{X}^{(\mathrm{Adm})} \cap\left\{m_{\max }+1, \ldots\right\}=\emptyset$.

Corollary 8 Let $l \in \mathbb{N}, c_{m} \in \mathbb{N}, w_{d} \in \mathbb{R}^{+}$and $r_{d} \in \mathbb{R}^{+}$then:

$$
\begin{aligned}
& O p t_{\infty}\left(l, c_{m}, r_{d}, w_{d}\right)= \\
& \min _{1 \leq j \leq m_{\max }}\left\{\begin{array}{l}
O p t_{0}\left(m-1, c_{m}\right) \\
w_{d}+j u_{f}+O p t_{\infty}\left(m-1-j, c_{m}, r_{d}, w_{d}\right)+r_{d}+O p t_{1}^{(d)}\left(j-1, c_{m}, r_{d}\right)
\end{array}\right.
\end{aligned}
$$

Proof. This is a direct corollary from Lemma 8

Note that as for Corollary 7, Corollary 8 is not directly involved in the proof of Theorem 2, but provides a faster execution of $\mathrm{Opt}_{\infty}\left(l, c_{m}, w_{d}, r_{d}\right)$.

\subsection{Construction of $\mathcal{M}_{X}$ and periodicity}

In the previous section, we proved that, for a given platform (defined by its parameters $c_{m}, w_{d}$, and $r_{d}$ ) the set of admissible periods that can be used by a DOS is finite. But this set can be quite large in practice. In this section, we define a sufficient subset $\mathcal{M}_{X}$ of $\mathcal{M}_{X}^{(\mathrm{Adm})}$ and highlight its element $m_{X}$ such that for any adjoint computation chain of any size, there is an optimal solution with only periods of size $m_{X}$, except for a bounded number of them. We also provide, in this section, an algorithm to compute $\mathcal{M}_{X}$ and $m_{X}$.

\subsubsection{Bounding the number of optimal solutions with less than one period}

Definition $10\left(\mathcal{N}_{X}(l), l_{X}^{(1)}, l_{X}^{(2)}\right)$ Let $\mathcal{N}_{X}(l)$ the largest (by inclusion) set such that for all $n_{l} \in \mathcal{N}_{X}(l)$, there exists a DOS for $\operatorname{PROB}_{\infty}\left(l, c_{m}, w_{d}, r_{d}\right)$ with $n_{l}$ periods.

Let $l_{X}^{(1)}$ be the smallest integer such that for all $l \geq l_{X}^{(1)}, \mathcal{N}_{X}(l) \not \subset\{0\}$, that is, the smallest integer such that for all chain sizes greater than $l_{X}^{(1)}$, there exists a DOS with at least one period.

Let $l_{X}^{(2)}$ be the smallest integer such that for all $l \geq l_{X}^{(2)}, \mathcal{N}_{X}(l) \not \subset\{0,1\}$, that is, the smallest integer such that for all chain sizes greater than $l_{X}^{(2)}$, there exists a DOS with at least two periods.

Lemma 9 Let $l \in \mathbb{N}$, then

$$
\mathcal{N}_{X}(l) \not \subset\{0\} \Longrightarrow \mathcal{N}_{X}(l+1) \not \subset\{0\} .
$$

Proof. Consider $l \in \mathbb{N}$, such that $\mathcal{N}_{X}(l) \not \subset\{0\}$. Then, there is a $n_{l} \in \mathcal{N}_{X}(l)$ such that $n_{l} \geq 1$. Let $\mathcal{S}_{l}=\left(m_{1}, \cdots, m_{n_{l}} ;\right.$ res $)$ be a DOS for $\operatorname{PROB}_{\infty}\left(l, c_{m}, w_{d}, r_{d}\right) . \mathcal{S}_{l}^{\prime}=(; l)$ is a DS for $\operatorname{PROB}_{\infty}\left(l, c_{m}, w_{d}, r_{d}\right)$. Thus by definition,

$$
\mathcal{E} x e c\left(\mathcal{S}_{l}\right) \leq \mathcal{E} x e c\left(\mathcal{S}_{l}^{\prime}\right)
$$

Let $\mathcal{S}_{l+1}^{\prime}=(; l+1)$ and $\mathcal{S}_{l+1}=\left(m_{1}, \cdots, m_{n_{X_{n}}(l)} ;\right.$ res +1$)$. They are both DS for 
$\operatorname{PROB}_{\infty}\left(l+1, c_{m}, w_{d}, r_{d}\right)$. Then we have:

$$
\begin{aligned}
\mathcal{E} x e c & \left.\mathcal{S}_{l+1}\right)-\mathcal{E} x e c\left(\mathcal{S}_{l+1}^{\prime}\right) \\
& \leq \mathcal{E} x e c\left(\mathcal{S}_{l+1}\right)-\mathcal{E} x e c\left(\mathcal{S}_{l+1}^{\prime}\right)+\left(\mathcal{E} x e c\left(\mathcal{S}_{l}^{\prime}\right)-\mathcal{E} x e c\left(\mathcal{S}_{l}\right)\right) \\
& =\left(\mathcal{E} x e c\left(\mathcal{S}_{l+1}\right)-\mathcal{E} x e c\left(\mathcal{S}_{l}\right)\right)-\left(\mathcal{E} x e c\left(\mathcal{S}_{l+1}^{\prime}\right)-\mathcal{E} x e c\left(\mathcal{S}_{l}^{\prime}\right)\right) \\
& =\left(\operatorname{Opt}_{0}\left(\operatorname{res}+1, c_{m}\right)-\operatorname{Opt}_{0}\left(\operatorname{res}, c_{m}\right)\right)-\left(\operatorname{Opt}_{0}\left(l+1, c_{m}\right)-\operatorname{Opt}_{0}\left(l, c_{m}\right)\right) \\
& \leq 0
\end{aligned}
$$

Equation $19 \mathrm{~b}$ is because $\mathcal{E} x e c\left(\mathcal{S}_{l}\right) \leq \mathcal{E} x e c\left(\mathcal{S}_{l}^{\prime}\right)$. Then we obtain Equation 19c through Equation (14). Finally, Equation (19d) is because $l \mapsto \operatorname{Opt}_{0}\left(l, c_{m}\right)$ is convex (Lemma 2$)$.

Finally, if $\mathcal{S}_{l+1}^{\prime}$ is a DOS then $\mathcal{S}_{l+1}$ is also a DOS. Hence, either $0 \notin \mathcal{N}_{X}(l+1)$ or $n_{l} \in \mathcal{N}_{X}(l+1)$. In both cases, $\mathcal{N}_{X}(l+1) \not \subset\{0\}$.

Corollary 9 For all $l \in \mathbb{N}$ :

$$
l<l_{X}^{(1)} \Longrightarrow \mathcal{N}_{X}(l)=\{0\}
$$

Proof. This is a corollary of Lemma 9 .

Lemma 10 (Existence of $l_{X}^{(1)}$ ) There exists $l>0$, such that $0 \notin \mathcal{N}_{X}(l)$. Furthermore,

$$
l_{X}^{(1)} \leq \beta\left(c_{m}, t_{d}+3\right)-\frac{\beta\left(c_{m}, t_{d}+2\right)}{2},
$$

where $t_{d}$ is the unique integer satisfying $\beta\left(c_{m}, t_{d}-1\right)<\frac{2\left(w_{d}+r_{d}\right)}{u_{f}} \leq \beta\left(c_{m}, t_{d}\right)$.

Proof. Given $j \geq \beta\left(c_{m}, t_{d}+1\right)$, let us show that

$$
\operatorname{Opt}_{0}\left(j, c_{m}\right)>\min _{k<j}\left(w_{d}+r_{d}+\mathrm{Opt}_{1}^{(d)}\left(k-1, c_{m}, r_{d}\right)+w_{d}+r_{d}+\mathrm{Opt}_{1}^{(d)}\left(j-k, c_{m}, r_{d}\right)\right)
$$

According to Theorem 4. there exist $\tilde{j}$ such that: (i) $\beta\left(c_{m}, t_{d}\right) \leq \tilde{j}$, and (ii) $\mathrm{Opt}_{0}\left(j, c_{m}\right)=\tilde{j} u_{f}+\operatorname{Opt}_{0}\left(\tilde{j}-1, c_{m}\right)+\operatorname{Opt}_{0}\left(j-\tilde{j}, c_{m}\right)$. Then,

$$
\begin{aligned}
\mathrm{Opt}_{0}\left(j, c_{m}\right) & =\tilde{j} u_{f}+\mathrm{Opt}_{0}\left(\tilde{j}-1, c_{m}\right)+\mathrm{Opt}_{0}\left(j-\tilde{j}, c_{m}-1\right) \\
& \geq \beta\left(c_{m}, t_{d}\right) u_{f}+\mathrm{Opt}_{0}\left(\tilde{j}-1, c_{m}\right)+\mathrm{Opt}_{0}\left(j-\tilde{j}, c_{m}\right) \\
& >2\left(w_{d}+r_{d}\right)+\mathrm{Opt}_{1}^{(d)}\left(\tilde{j}-1, c_{m}, r_{d}\right)+\mathrm{Opt}_{1}^{(d)}\left(j-\tilde{j}, c_{m}, r_{d}\right)
\end{aligned}
$$

which shows Equation (21) (by taking the minimum).

Let us now consider $l \geq \beta\left(c_{m}, t_{d}+3\right)$, then according to Theorem 4 , there exist $j$ such that: (i) $\beta\left(c_{m}, t_{d}+2\right) \leq j$, and (ii) $\operatorname{Opt}_{0}\left(l, c_{m}\right)=j u_{f}+\mathrm{Opt}_{0}\left(j-1, c_{m}\right)+\mathrm{Opt}_{0}\left(l-j, c_{m}-1\right)$.

Let $m_{1}, m_{2}$ such that $m_{1}+m_{2}=j$ and

$$
\begin{aligned}
\mathrm{Opt}_{1}^{(d)}\left(m_{1}-1, c_{m}, r_{d}\right) & +\mathrm{Opt}_{1}^{(d)}\left(m_{2}-1, c_{m}, r_{d}\right) \\
& =\min _{k<j-1} \mathrm{Opt}_{1}^{(d)}\left(k-1, c_{m}, r_{d}\right)+\mathrm{Opt}_{1}^{(d)}\left(j-1-k, c_{m}, r_{d}\right) .
\end{aligned}
$$


Clearly, $\left(m_{1}, m_{2} ; l-j\right)$ is a DS for $\operatorname{PROB}_{\infty}\left(l, c_{m}, w_{d}, r_{d}\right)$. Let us now show that $\mathcal{E x e c}\left(m_{1}, m_{2} ; l-j\right)<\mathcal{E} x e c(; l)$ which shows the result.

$$
\begin{aligned}
\mathcal{E x e c}\left(m_{1}, m_{2} ; l-j\right) & =j u_{f}+\operatorname{Opt}_{0}\left(l-j, c_{m}\right)+\sum_{i=1}^{2}\left(w_{d}+r_{d}+\mathrm{Opt}_{1}^{(d)}\left(m_{i}-1, c_{m}, r_{d}\right)\right) \\
& \leq j u_{f}+\mathrm{Opt}_{0}\left(l-j, c_{m}-1\right)+\sum_{i=1}^{2}\left(w_{d}+r_{d}+\mathrm{Opt}_{1}^{(d)}\left(m_{i}-1, c_{m}, r_{d}\right)\right) \\
& <j u_{f}+\operatorname{Opt}_{0}\left(l-j, c_{m}-1\right)+\mathrm{Opt}_{0}\left(j-1, c_{m}\right) \\
& <\mathcal{E x e c}(; l)
\end{aligned}
$$

Equation (22a) is due to the fact that adding memory checkpoints can only improve the execution time, Equation (22b) is because of Equation (21).

Hence the result: for $l \geq \beta\left(c_{m}, t_{d}+3\right), 0 \notin \mathcal{N}_{X}(l)$. Corollary 9 then gives us that $l \geq l_{X}^{(1)}$, hence the result: $l_{X}^{(1)} \leq \beta\left(c_{m}, t_{d}+3\right)$. We can improve this result by saying that $\left(m_{1} ; l-j\right)$ (resp. $\left.\left(m_{2} ; l-j\right)\right)$ is a DOS for $\operatorname{PROB}_{\infty}\left(l-m_{2}, c_{m}, w_{d}, r_{d}\right)\left(\right.$ resp. $\left.\mathrm{PROB}_{\infty}\left(l-m_{1}, c_{m}, w_{d}, r_{d}\right)\right)$ by Corollary 5. Furthermore, by Lemma 9, this implies that for all $l \geq \beta\left(c_{m}, t_{d}+3\right)-$ $\max \left(m_{1}+m_{2}\right), 0 \notin \mathcal{N}_{X}(l)$. Hence, $l_{X}^{(1)} \leq \beta\left(c_{m}, t_{d}+3\right)-\max \left(m_{1}+m_{2}\right)$. Furthermore, we have seen that $m_{1}+m_{2} \geq \beta\left(c_{m}, t_{d}+2\right)$ and in particular, $\max \left(m_{1}+m_{2}\right) \geq \frac{\beta\left(c_{m}, t_{d}+2\right)}{2}$.

Finally, we have the result,

$$
l_{X}^{(1)} \leq \beta\left(c_{m}, t_{d}+3\right)-\frac{\beta\left(c_{m}, t_{d}+2\right)}{2} .
$$

Based on Corollary 9 and Lemma 10 we can construct an algorithm to compute $l_{X}^{(1)}$. Corollary 9 says that Algorithm 3 returns the value of $l_{X}^{(1)}$, while Lemma 10 ensures that the algorithm terminates in less than $\beta\left(c_{m}, t_{d}+3\right)-\frac{\beta\left(c_{m}, t_{d}+2\right)}{2}$ iterations of the "while" loop.

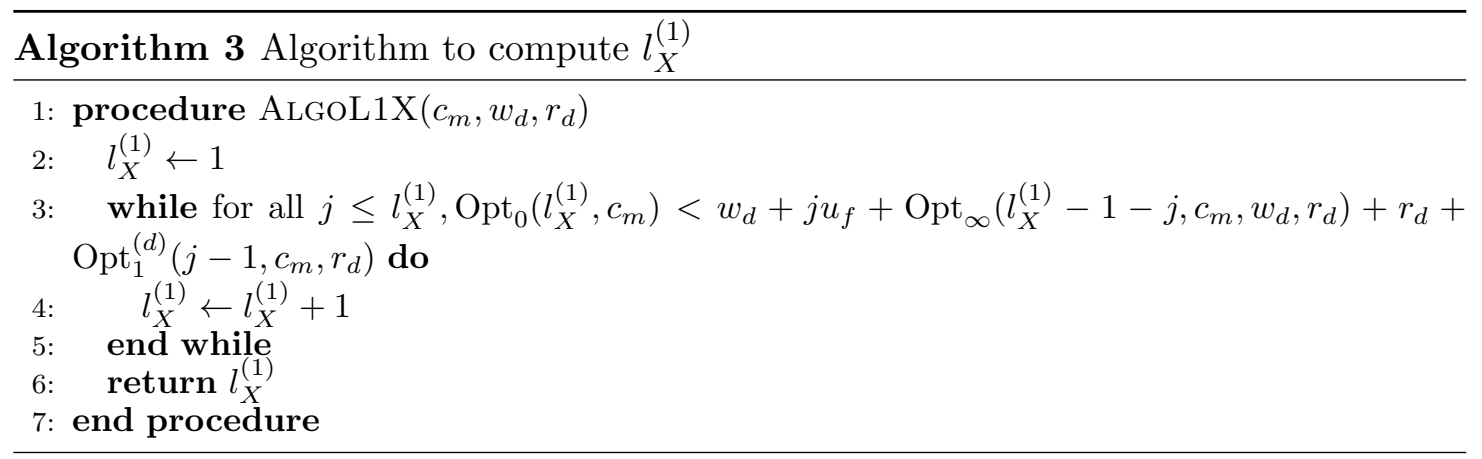

We can now confirm the existence of $l_{X}^{(2)}$ defined in Definition 10 .

LEMMA 11 (Existence of $l_{X}^{(2)}$ ) There exists a smallest integer $l_{X}^{(2)}$ such that for all chain 
sizes greater than $l_{X}^{(2)}$, there exists a DOS with at least two periods; and

$$
l_{X}^{(2)} \leq l_{X}^{(1)}+m_{\max },
$$

where $m_{\max }$ is defined by Equation (18).

Proof. Let $l \geq l_{X}^{(1)}$. By definition, we have $\mathcal{N}_{X}(l) \not \subset\{0\}$. Furthermore, let us show that for all $l \geq l_{X}^{(1)}+m_{\max }, \mathcal{N}_{X}(l) \not \subset\{0,1\}$.

Let $l$ such that $1 \in \mathcal{N}_{X}(l)$ and $\mathcal{N}_{X}(l) \subset\{0,1\}$. Let $(m ; l-m)$ be a DOS for $\operatorname{PROB}_{\infty}\left(l, c_{m}, w_{d}, r_{d}\right)$. According to Lemma 8, $m \leq m_{\text {max }}$. Furthermore, according to Corollary $5, \mathcal{N}_{X}(l-m)=\{0\}$. Hence, according to Lemma 9, $l-m<l_{X}^{(1)}$. Finally, we have: $l<l_{X}^{(1)}+m_{\max }$.

\subsubsection{M-DOS and periodicity}

In order to show the periodicity, we define a set $\mathcal{M}_{X}$ such that for all $l$, there exists a DOS for $\operatorname{PROB}_{\infty}\left(l, c_{m}, w_{d}, r_{d}\right)$ such that all its periods are in $\mathcal{M}_{X}$. We call such DOS: M-DOS.

Definition $11\left(\mathcal{M}_{X}, m_{X}\right)$ For this section we use the following definitions:

- Let $\mathcal{L}_{X}$ be the set of adjoint chains that only admits DOS with one period:

$$
\mathcal{L}_{X}=\left\{l \mid \mathcal{N}_{X}(l)=\{1\}\right\}
$$

- We define the relative cost of a period ${ }^{3} \operatorname{RelCost} X$ :

$$
\operatorname{RelCost}_{X}: m \mapsto \frac{w_{d}+r_{d}+\mathrm{Opt}_{1}^{(d)}\left(m-1, c_{m}, r_{d}\right)}{m} .
$$

- For $l \in \mathcal{L}_{X}$, we define $m_{1}^{l}$ to be the minimum element of $\left\{m \mid(m ; l-m)\right.$ is a DOS for $\left.\operatorname{PROB}_{\infty}\left(l, c_{m}, w_{d}, r_{d}\right)\right\}$ with regard to RelCost ${ }_{X}$.

- We define a subset of the set of admissible periods $\mathcal{M}_{X}$ :

$$
\mathcal{M}_{X}=\left\{m_{1}^{l} \mid l \in \mathcal{L}_{X}\right\}
$$

- Denote $m_{X}$ the largest element of $\mathcal{M}_{X}$ that is minimum with regard to RelCost . $_{\text {. }}$

Note that according to Corollary 9, $\mathcal{L}_{X} \subset\left\{l_{X}^{(1)}, \cdots, \infty\right\}$, and by Definition $10, \mathcal{L}_{X} \subset$ $\left\{1, \cdots, l_{X}^{(2)}-1\right\}$. Hence

$$
\left|\mathcal{M}_{X}\right|<l_{X}^{(2)}-l_{X}^{(1)}
$$

Corollary 9 provides an efficient way to check whether $l \in \mathcal{L}_{X}$ :

\footnotetext{
${ }^{3}$ Intuitively, for a period of size $m$ we pay $w_{d}$ to be able to execute it later, and then $r_{d}+\mathrm{Opt}_{1}^{(d)}\left(m-1, c_{m}, w_{d}, r_{d}\right)$ to actually execute it.
} 
Proposition $1 \quad l \in \mathcal{L}_{X}$ if and only if

$O p t_{\infty}\left(l, c_{m}, w_{d}, r_{d}\right)<\min _{j \leq l-l_{X}^{(1)}}\left\{\begin{array}{l}O p t_{0}\left(l, c_{m}\right) \\ w_{d}+j u_{f}+O p t_{\infty}\left(l-1-j, c_{m}, r_{d}, w_{d}\right)+r_{d}+O p t_{1}^{(d)}\left(j-1, c_{m}, r_{d}\right)\end{array}\right.$

Proof. If $l \in \mathcal{L}_{X}$ then $\operatorname{Opt}_{\infty}\left(l, c_{m}, r_{d}, w_{d}\right)<\operatorname{Opt}_{0}\left(l, c_{m}\right)$ (because $\left.0 \notin \mathcal{N}_{X}(l)\right)$. Furthermore, by Corollary 9, if a DOS for $\operatorname{PROB}_{\infty}\left(l, c_{m}, w_{d}, r_{d}\right)$ admits a period $m$ such that $l-m \geq l_{X}^{(1)}$, then according to Corollary 5. $\mathcal{N}_{X}(l-m) \neq\{0\}$ and $l \notin \mathcal{L}_{X}$ (if $n_{l-m} \in \mathcal{N}_{X}(l-m), n_{l-m}+1 \in \mathcal{N}_{X}(l)$ because $m$ is a period of a DOS of $\left.\operatorname{PROB}_{\infty}\left(l, c_{m}, w_{d}, r_{d}\right)\right)$.

Finally, if $\mathrm{Opt}_{0}\left(l, c_{m}\right)$ satisfies Equation (27), then $\mathrm{Opt}_{\infty}\left(l, c_{m}, w_{d}, r_{d}\right)=w_{d}+j u_{f}+$ $\mathrm{Opt}_{\infty}\left(l-1-j, c_{m}, r_{d}, w_{d}\right)+r_{d}+\mathrm{Opt}_{1}^{(d)}\left(j-1, c_{m}, r_{d}\right)$ only for $j$ such that $l-j<l_{X}^{(1)}$, and by Corollary $9, \mathcal{N}_{X}(l)=\{1\}$.

Proposition 1 in turn provides an efficient way to compute $m_{X}$ :

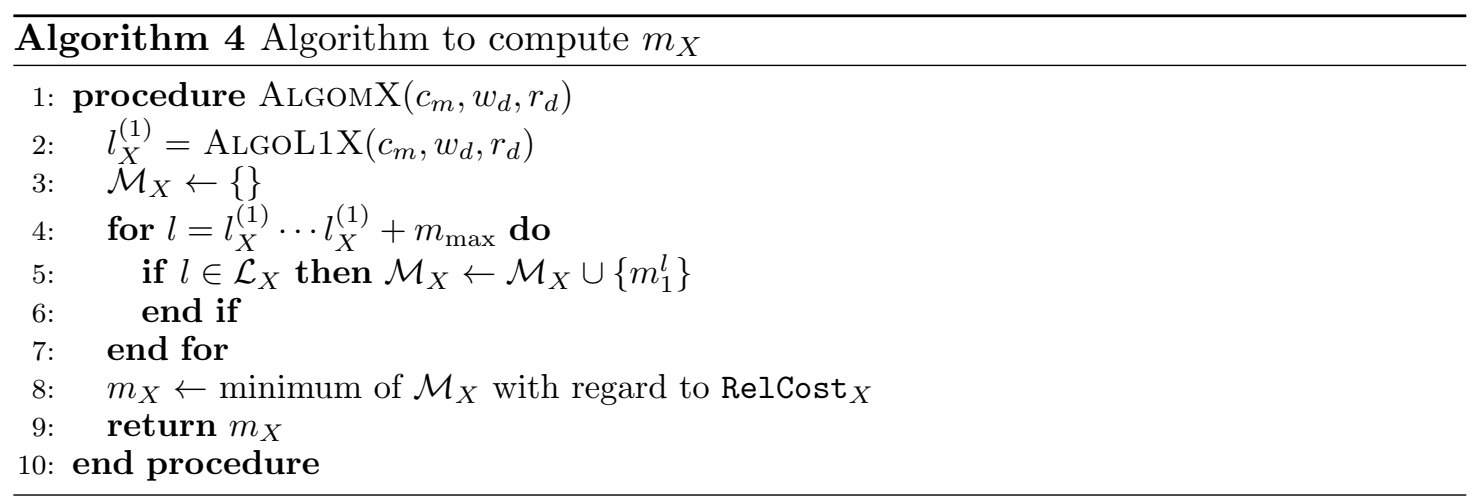

Definition 12 (M-DOS) For all $l, \mathcal{S}=\left(m_{1}, \cdots, m_{n_{l}}\right.$;res) is a M-DOS for $\operatorname{PROB}_{\infty}\left(l, c_{m}, w_{d}, r_{d}\right)$ if (i) $\mathcal{S}$ is a DOS and (ii), for all $i, m_{i} \in \mathcal{M}_{X}$.

TheOREm 9 (Existence of M-DOS) Given $X=\left(c_{m}, r_{d}, w_{d}\right)$. For all $l \geq l_{X}^{(1)}$, there exists a $M-D O S$.

Proof. We show the result by contradiction.

Let us call $l_{0} \geq l_{X}^{(1)}$ the minimum length that does not admit a M-DOS, i.e., such that for all DOS for $\mathrm{PROB}_{\infty}\left(l_{0}, c_{m}, w_{d}, r_{d}\right)$ there exists a period not in $\mathcal{M}_{X}$.

Amongst the DOS for $\operatorname{PROB}_{\infty}\left(l_{0}, c_{m}, w_{d}, r_{d}\right)$ such that the number of periods is greater than 2 (by definition $l_{0} \geq l_{X}^{(1)}$, and $l_{0} \notin \mathcal{L}_{X}$ hence there exists at least one), we choose $\mathcal{S}=\left(m_{1}, \cdots, m_{n_{l_{0}}}\right.$; res $)$ a DOS that is minimum with regard to the number of periods not in $\mathcal{M}_{X}$.

By Corollary 5 (item (1) $)$ we can further assume w.l.o.g that $m_{n_{l_{0}}} \notin \mathcal{M}_{X}$. Iterating Corollary 5 (item $(2)),\left(m_{n_{l_{0}}} ;\right.$ res) is a DOS for $\mathrm{Opt}_{\infty}\left(m_{n_{l_{0}}}+\right.$ res, $\left.c_{m}, w_{d}, r_{d}\right)$.

Clearly, $m_{n_{l_{0}}}+$ res $<l_{0}$ (there are more than two periods, hence $l_{0} \geq m_{1}+m_{n_{l_{0}}}+$ res $>m_{n_{l_{0}}}+$ res), and because $1 \in \mathcal{N}_{X}\left(m_{n_{l_{0}}}+\right.$ res), by Lemma $9, m_{n_{l_{0}}}+$ res $\geq l_{X}^{(1)}$. By minimality of $l_{0}$, there exists $\mathcal{S}^{\prime}$ a DOS for $\operatorname{Opt}_{\infty}\left(m_{n_{l_{0}}}+\right.$ res, $\left.c_{m}, w_{d}, r_{d}\right)$ such that all periods of $\mathcal{S}^{\prime}$ are in $\mathcal{M}_{X}$ and such that there is at least one period.

Finally, by Corollary 5 (item $(3))$, we can replace $\left(m_{n_{l_{0}}} ;\right.$ res) in $\mathcal{S}$ by $\mathcal{S}^{\prime}$, then (i) it will still be an optimal algorithm for $l_{0}$, and (ii) it will have more than 2 periods. 
Finally, we have a DOS for $\mathrm{PROB}_{\infty}\left(l_{0}, c_{m}, w_{d}, r_{d}\right)$ with more than two periods and one less period than $\mathcal{S}$ not in $\mathcal{M}_{X}$ contradicting the existence of $\mathcal{S}$.

Definition $13\left(M_{-m_{X}}^{\mathcal{S}}\right)$ Let $\mathcal{S}=\left(m_{1}, \cdots, m_{n_{l}} ;\right.$ res $)$ be a M-DOS for $\operatorname{PROB}_{\infty}\left(l, c_{m}, w_{d}, r_{d}\right)$, we define

$$
M_{-m_{X}}^{\mathcal{S}}=\sum_{i \in\left\{i \mid m_{i} \neq m_{X}\right\}} m_{i}
$$

Less formally, $M_{-m_{X}}^{\mathcal{S}}$ is the sum of the length of the periods of $\mathcal{S}$ that are not $m_{X}$.

Lemma 12 For all $l \in \mathbb{N}$, there exists $\mathcal{S}$ a $M$-DOS for $\operatorname{PROB}_{\infty}\left(l, c_{m}, w_{d}, r_{d}\right)$ such that, for all $m \in \mathcal{M}_{X} \backslash\left\{m_{X}\right\}$, there are less than $m_{X}$ periods of size $m$.

Proof. We show the result by contradiction. Let $l$ such that for any given M-DOS for $\operatorname{PROB}_{\infty}\left(l, c_{m}, w_{d}, r_{d}\right)$, there exists $m \in \mathcal{M}_{X} \backslash\left\{m_{X}\right\}$ and there are not less than $m_{X}$ periods of size $m$. Let $\mathcal{S}=\left(m_{1}, \cdots, m_{n_{l}}\right.$; res) a M-DOS for $\operatorname{PROB}_{\infty}\left(l, c_{m}, w_{d}, r_{d}\right)$ that is minimal with regards to the function $\mathcal{S} \mapsto M_{-m_{X}}^{\mathcal{S}}$. Let $m \in \mathcal{M}_{X} \backslash\left\{m_{X}\right\}$ such that there are not less than $m_{X}$ periods of size $m$ in $\mathcal{S}$. Without loss of generality, we can assume that for $i \leq m_{X}, m_{i}=m$ (Corollary 5 (item (1)), the $m_{X}$ first periods have a size $m$ ).

According to Corollary 3 (and by induction on the $m_{X}$ first elements),

$$
\mathcal{E} x e c(\mathcal{S})=m_{X}\left(m u_{f}+w_{d}+r_{d}+\mathrm{Opt}_{1}^{(d)}\left(m-1, c_{m}, r_{d}\right)\right)+\mathcal{E} x e c\left(m_{m_{X}+1}, \ldots, m_{n_{l}} ; \text { res }\right)
$$

Denote $\mathcal{S}^{\prime}=\left(m_{1}^{\prime}, \cdots, m_{m}^{\prime}, m_{m_{X}+1}, \cdots, m_{n_{l}}\right.$; res $)$, where for $i \leq m, m_{i}^{\prime}=m_{X}$. Then clearly, $\sum_{i=1}^{m} m_{X}=\sum_{i=1}^{m_{X}} m$, and then $\mathcal{S}^{\prime}$ is a DS for $\operatorname{PROB}_{\infty}\left(l, c_{m}, w_{d}, r_{d}\right)$.

Furthermore,

$\mathcal{E} x e c\left(\mathcal{S}^{\prime}\right)=m\left(m_{X} u_{f}+w_{d}+r_{d}+\operatorname{Opt}_{1}^{(d)}\left(m_{X}-1, c_{m}, r_{d}\right)\right)+\mathcal{E} x e c\left(m_{m_{X}+1}, \ldots, m_{n_{l}} ;\right.$ res $)$

Then,

$$
\begin{aligned}
& \mathcal{E x e c}(\mathcal{S})-\mathcal{E x e c}\left(\mathcal{S}^{\prime}\right)= \\
& \quad m_{X}\left(w_{d}+r_{d}+\mathrm{Opt}_{1}^{(d)}\left(m-1, c_{m}, r_{d}\right)\right)-m\left(w_{d}+r_{d}+\mathrm{Opt}_{1}^{(d)}\left(m_{X}-1, c_{m}, r_{d}\right)\right)
\end{aligned}
$$

Hence, because $m_{X}$ is minimal with regards to the function $\operatorname{RelCost}{ }_{X}, \operatorname{RelCost} \operatorname{Cos}_{X}(m) \geq$ $\operatorname{RelCost}_{X}\left(m_{X}\right)$ and $\mathcal{E} x e c(\mathcal{S})-\mathcal{E} x e c\left(\mathcal{S}^{\prime}\right) \geq 0$.

By optimality of $\mathcal{S}$, we have: $\mathcal{E} x e c(\mathcal{S})=\mathcal{E} x e c\left(\mathcal{S}^{\prime}\right)$. However

$$
M_{-m_{X}}^{\mathcal{S}^{\prime}}=M_{-m_{X}}^{\mathcal{S}}-m_{X} \cdot m,
$$

which contradicts the minimality of $\mathcal{S}$, proving the result.

Proof of Theorem 2. Let:

$$
\begin{aligned}
& i_{X}^{\mathrm{ub}}=\left(m_{X}-1\right) \cdot\left(\left|\mathcal{M}_{X}\right|-1\right) \\
& l_{X}^{\mathrm{ub}}=l_{X}^{(1)}+i_{X}^{\mathrm{ub}} m_{\max } .
\end{aligned}
$$



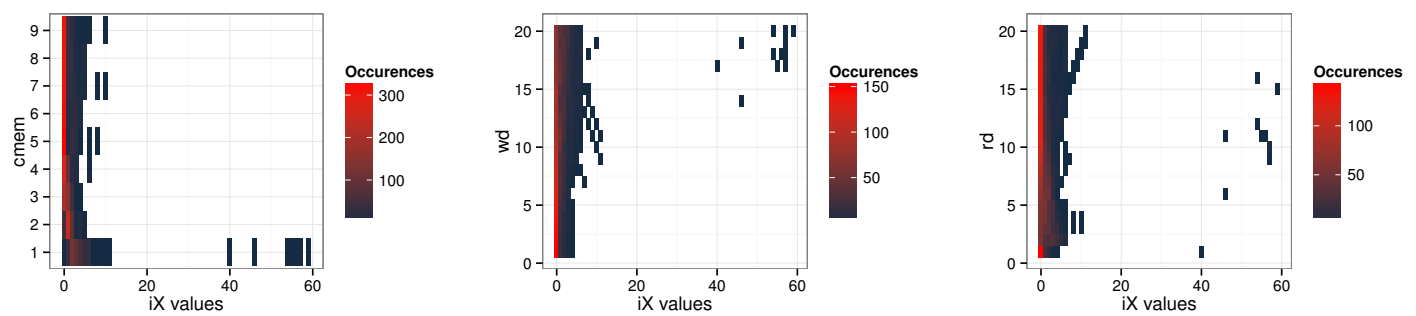

Figure 3.: Heat maps of the values of $i_{X}$ according to the three different platform parameters: $c_{m}, w_{d}, r_{d}$ (white $=0$ occurence). Most of the time, $i_{X} \leq 10$.

Let $l \geq l_{X}^{\mathrm{ub}}$, then according to Theorem 9 and Lemma 12 , we can construct $\mathcal{S}=$ $\left(m_{1}, \cdots, m_{n_{l}} ;\right.$ res $)$ a M-DOS for $\operatorname{PROB}_{\infty}\left(l, c_{m}, w_{d}, r_{d}\right)$ such that for any $m \in \mathcal{M}_{X} \backslash\left\{m_{X}\right\}$, at most $m_{X}-1$ periods of size $m$. Hence, there are at most $i_{X}^{\text {ub }}$ periods not equal to $m_{X}$, and $n_{l}-i_{X}^{\mathrm{ub}}$ periods of size $m_{X}$.

According to Corollary 5 (item (1) , we can assume that the first $n_{l}-i_{X}^{\mathrm{ub}}$ periods are the periods of size $m_{X}$ (hence showing Equation (3p).

Furthermore, according to Corollary 5 (item $(2)$,

- $\left(m_{n_{l}-i_{X}^{\mathrm{ub}}}, \cdots, m_{n_{l}} ;\right.$ res $)$ is a M-DOS with $i_{X}^{\mathrm{ub}}$ periods all smaller than $m_{\max }$;

- $($;res $)$ is a DOS hence res $<l_{X}^{(1)}$.

Finally, $\sum_{i=n_{l}-i_{X}^{\mathrm{ub}}}^{n_{l}} m_{i}+$ res $<l_{X}^{\mathrm{ub}}$ (hence showing Equation (4)).

This shows the existence of $i_{X}$ and $l_{X}$.

In this proof, we have not given the smallest value possible for $i_{X}$ and $l_{X}$, we have only given upper bounds to show their existence.

\subsection{Experimental evaluation of $i_{X}$ and $m_{X}$}

Theorem 2 only gives a weak periodicity argument. In this section we try to develop questions that can arise from Theorem 2.

\subsubsection{Can we get a better bound for $i_{X}$ ?}

One might expect that by improving the bounds obtained in the various results of the previous sections, one may be able to show periodicity or be able to give a bound on $i_{X}$ that does not depend on $X$. Unfortunately, there is little chance of this result being true. We studied for all triplet $\left(c_{m}, w_{d}, r_{d}\right) \in\{1, \ldots, 9\} \times\{1, \ldots, 20\} \times\{1, \ldots, 20\}$ the value of $i_{X}$ (by computing the optimal solutions of every value $l \in\left\{1, \ldots, l_{X}\right\}$ ) and reported it in Figure 3.

An important observation from Figure 3 is that (i) most of the time $i_{X}$ is very small (0 or 1 ), and (ii) $i_{X}$ is highly influenced by $c_{m}$, for instance when $c_{m} \geq 2$, we have never obtained values for $i_{X}$ greater than 10. On the contrary, when $c_{m}=1$ and $w_{d}$ is large, then $i_{X}$ can take large values (we have observed up to 59). In general, $i_{X}$ is small, for instance $59 \%$ of the time $i_{X}$ is equal to $0,93 \%$ of the time $i_{X}$ is between 0 and 3 .

\subsubsection{Can we compute $m_{X}$ ?}

The second question that can be raised is about the value of $m_{X}$. Unfortunately we have been unable to prove an analytical formula for $m_{X}$, this remains an open question. 
However by studying values of $m_{X}$ for small values of $c_{m}, w_{d}$ and $r_{d}$, we have conjectured an analytical formula for $m_{X}$. We have then verified it on a larger set $\left(\left(c_{m}, w_{d}, r_{d}\right) \in\right.$ $\{1, \ldots, 10\} \times\{1, \ldots, 50\} \times\{1, \ldots, 50\})$ without finding any counter-examples.

ConjeCture 1 We define $f$ a function that takes three integers $x, y$ and $c_{m}$ as follow:

$$
f:\left(x, y, c_{m}\right) \mapsto \beta\left(c_{m}+1, x+y-1\right)-\sum_{k=0}^{y-1} \beta\left(c_{m}, k\right) .
$$

Let $i_{r}\left(r_{d}, c_{m}\right)=x$ or $x+1$, where $x$ is the only integer that satisfies

$$
\beta\left(c_{m}+1, x-1\right) \leq r_{d}<\beta\left(c_{m}+1, x\right) .
$$

Let $i_{w}\left(w_{d}, r_{d}, c_{m}\right)=y$, where $y$ is the only integer that satisfies

$$
\sum_{j=1}^{y-1} f\left(j, i_{r}\left(r_{d}, c_{m}\right), c_{m}\right)<w_{d} \leq \sum_{j=1}^{i_{w}} f\left(j, i_{r}\left(r_{d}, c_{m}\right), c_{m}\right) .
$$

We conjecture that

$$
m_{X}=f\left(i_{r}\left(r_{d}, c_{m}\right), i_{w}\left(w_{d}, r_{d}, c_{m}\right), c_{m}\right) .
$$

Note that we have not been able to conjecture the exact value of $i_{r}\left(r_{d}, c_{m}\right)$, hence

\begin{tabular}{|c|c|c|c|c|c|c|c|c|c|c|c|}
\hline \multicolumn{7}{|c|}{$c_{m}=2$} & \multicolumn{5}{|c|}{$c_{m}=3$} \\
\hline$r_{d}$ & & & & $l_{X}$ & & & $r_{d}$ & & & $m_{X}$ & \\
\hline 1 & 3 & 9 & 19 & 34 & 55 & 83 & 1 & 4 & 14 & 34 & 125 \\
\hline 4 & 6 & 16 & 31 & 52 & 80 & 116 & 5 & 10 & 30 & 121 & \\
\hline 10 & 10 & 25 & 46 & 74 & & & 15 & 55 & 111 & & \\
\hline 20 & 15 & 36 & 64 & 100 & & & & & & & \\
\hline
\end{tabular}
leaving the possibility of two distinct periods $m_{X}$.

We give the example below that we used along with the online encyclopedia of integer sequences (OEIS) [7] to be able to do the conjecture.

Table 2.: Periods when $c_{m}=2$ (left) and $c_{m}=3$ (right) when $w_{d}$ increases, for given $r_{d}$. When $r_{d}$ is between two values (for instance for $r_{d}=3$ ), then $m_{X}$ will vary with $w_{d}$ between the values from the adjoint sequences $\left(r_{d}=1\right.$ and $r_{d}=4$ (resp. 5) for $c_{m}=2$ (resp. 3)).

\section{Asymptotically optimal online algorithm}

We now present an asymptotically optimal online algorithm DisK-A-REvolve to prove Corollary 1. Intuitively, this algorithm writes disk checkpoints periodically with a period of $m_{X}$.

Theorem 10 Disk-A-Revolve is asymptotically optimal for $\mathrm{PROB}_{\infty}\left(l, c_{m}, w_{d}, r_{d}\right)$. 


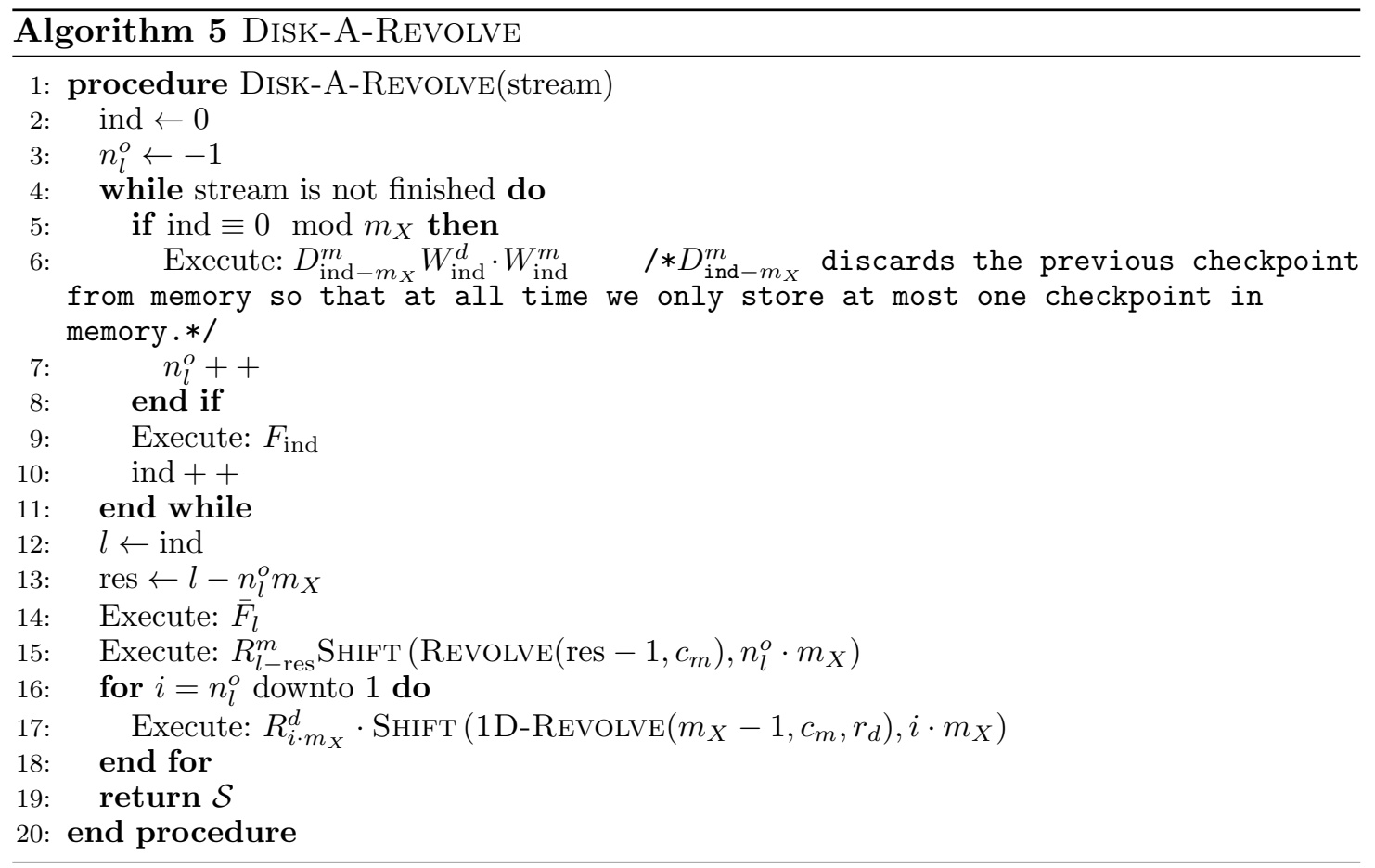

Proof. At the end of the while loop (line 4), $n_{l}^{o}=\left\lfloor l / m_{X}\right\rfloor-1$ (indeed, the "if" test (line 5] is executed $\left\lfloor l / m_{X}\right\rfloor$ times). The execution time of DisK-A-REvolve is:

- The while loop (line 4 ) does $n_{l}^{o}+1$ write to disks and $l$ forward steps.

- We then execute one backward operation, $\bar{F}_{l}$.

- The execution of Revolve then has a cost of $\mathrm{Opt}_{0}\left(\mathrm{res}-1, c_{m}\right)$

- Then the for loop (line 16 has a cost of $n_{l}^{o} \cdot\left(r_{d}+\mathrm{Opt}_{1}^{(d)}\left(m_{X}-1, c_{m}, r_{d}\right)\right.$ )

The total execution time is then:

$$
\begin{aligned}
& \mathcal{E x e c}(\text { Disk-A-Revolve }(\text { stream of size } l))= \\
& l u_{f}+\left(n_{l}^{o}+1\right) w_{d}+u_{b}+\mathrm{Opt}_{0}\left(\text { res }-1, c_{m}\right)+n_{l}^{o} \cdot\left(r_{d}+\mathrm{Opt}_{1}^{(d)}\left(m_{X}-1, c_{m}, r_{d}\right)\right)
\end{aligned}
$$

Then, asymptotically,

$$
\begin{aligned}
\mathcal{E} x e c(\text { Disk-A-Revolve(stream of size } l)) & \sim l u_{f}+n_{l}^{o}\left(w_{d}+r_{d}+\mathrm{Opt}_{1}^{(d)}\left(m_{X}-1, c_{m}, r_{d}\right)\right) \\
& \sim l u_{f}+\left(\left\lfloor l / m_{X}\right\rfloor-1\right)\left(w_{d}+r_{d}+\mathrm{Opt}_{1}^{(d)}\left(m_{X}-1, c_{m}, r_{d}\right)\right)
\end{aligned}
$$

According to Theorems 2 and 5 , the optimal execution time is greater than

$$
\left(l-l_{X}\right) u_{f}+\left\lfloor\frac{l-l_{X}}{m_{X}}\right\rfloor \cdot\left(w_{d}+r_{d}+\mathrm{Opt}_{1}^{(d)}\left(m_{X}-1, c_{m}, r_{d}\right)\right),
$$

which, in turn is equivalent to $l u_{f}\left(\left\lfloor l / m_{X}\right\rfloor-1\right)\left(w_{d}+r_{d}+\mathrm{Opt}_{1}^{(d)}\left(m_{X}-1, c_{m}, r_{d}\right)\right)$ asymptotically. Hence showing that DisK-A-REvolve is asymptotically optimal. 
Remark Note that the execution time of Disk-A-Revolve can still be improved by using efficiently the memory checkpoints and waiting until the last minute before storing data on disks. For instance, since $m_{X}$ is small, we can use in practice the optimal online algorithm designed by Stumm and Walter [9] for the memory checkpoints between disk checkpoints. However for readability reasons we chose not to present it here as it would not have changed the final result.

\section{Evaluation of a periodic schedule}

Using Theorem 2, we can easily compute a DOS for $\operatorname{PROB}_{\infty}\left(l, c_{m}, w_{d}, r_{d}\right)$. We need first to pre-compute a DOS for every adjoint chain of size smaller than $l_{X}$. Then, we can give in constant time a DOS for any $l$ using the following simple algorithm:

(1) Let $n_{1}=\left[\frac{l-l_{X}}{m_{X}}\right]$. Intuitively, $n_{1}$ is the number of periods that we are sure will be equal to $m_{X}$.

(2) Let $l^{\prime}=l-n_{1} \cdot m_{X}$. Intuitively, $l^{\prime}$ is the remainder of the work to be done. A DOS for $l^{\prime}$ has already been pre-computed: $\left(m_{1}, \cdots, m_{n_{2}} ;\right.$ res $)$.

(3) Then $\left(m_{X}, \ldots, m_{X}, m_{1}, \cdots, m_{n_{2}}\right.$; res) (with $n_{1}$ iterations of $m_{X}$ initially) is a DOS for $l$

This gives us a constant time algorithm for $\operatorname{PROB}_{\infty}\left(l, c_{m}, w_{d}, r_{d}\right)$. However, the precomputation part of this algorithm can be costly (in time and space) depending on the parameter of the platform.

\subsection{Periodic Dominant Schedules}

Based on the observation made in Section 4.7.1, that, in general, $i_{X}$ is small, we might be interested in only considering the Periodic Dominant Sequence, defined as follows:

Definition $14\left(\operatorname{PDS}\left(1, c_{m}, w_{d}, r_{d}\right)\right)$ Given values of $l, c_{m}, w_{d}$ and $r_{d}$, the Periodic Dominant Sequence PDS $\left(1, c_{m}, w_{d}, r_{d}\right)$ is the Dominant Sequence

$$
\operatorname{PDS}\left(1, c_{m}, w_{d}, r_{d}\right)=\left(m_{X}, m_{X}, \cdots, m_{X} ; \text { res }\right)
$$

where res $=\left\lceil\frac{l-l_{X}^{(1)}}{m_{X}}\right\rceil$.

The $\operatorname{PDS}\left(1, c_{m}, w_{d}, r_{d}\right)$ is not always optimal but it has the advantage of not requiring a costly pre-computation. It only needs the value of $m_{X}, l_{X}^{(1)}$ and the schedule 1D-Revolve $\left(m_{X}, c_{m}, r_{d}\right)$.

\subsection{Experimental Results}

In this section, we assess the time overhead of the Periodic Dominant Sequence and of Disk-A-Revolve compared with the optimal sequence that computes an Adjoint Computation chain of size $l$. In the experiments, we normalize every time values by setting $u_{f}=1$. Because the backward steps are computed exactly once in any solution, their cost has no impact on the algorithms: we set $u_{b}=0$ so that we can assess the performance of the algorithms on the forward sweep. Here we present results for $c_{m} \in\{5,10\}$ and $w_{d}=r_{d} \in\{1,2,5,10\}$. 


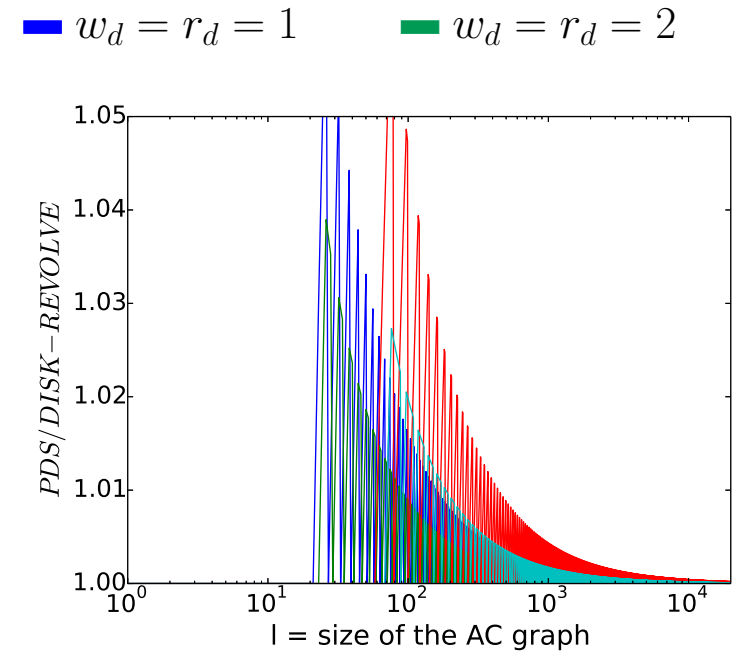

(a) $c_{m}=5$
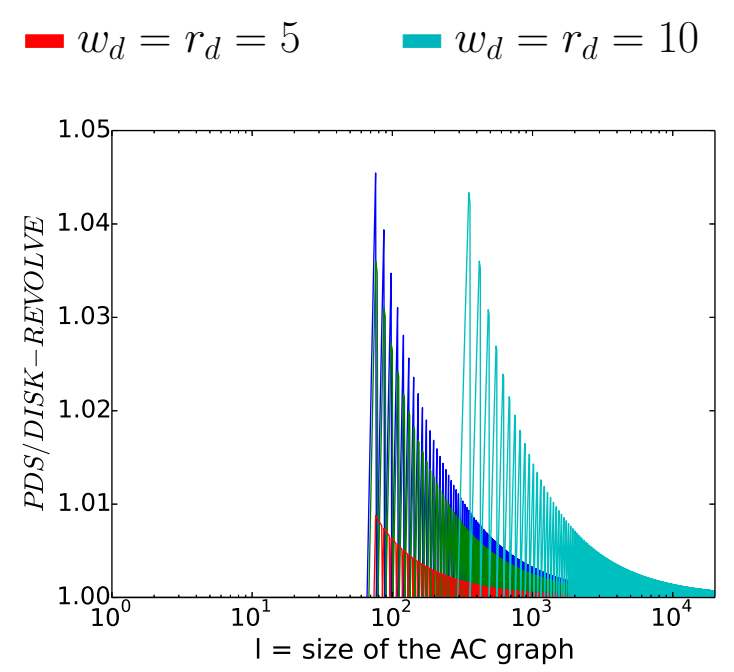

(b) $c_{m}=10$

Figure 4.: Makespan ratio of $\operatorname{PDS}\left(1, c_{m}, w_{d}, r_{d}\right) / \operatorname{DisK}-\operatorname{Revolve}\left(l, c_{m}, w_{d}, r_{d}\right)$ as a function of $l$.

Figure 4a and 4b depict the time overhead of $\operatorname{PDS}\left(1, c_{m}, w_{d}, r_{d}\right)$ compared with Disk-ReVOLVE $\left(l, c_{m}, w_{d}, r_{d}\right)$ as a function of $l$. We observe that the ratio increases with $w_{d}$ and $r_{d}$ but for large instances of the problem ( $l$ greater than 1000), the time overhead is always less than $1 \%$.

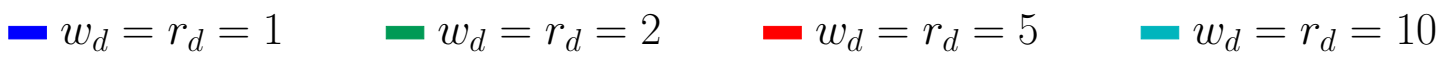

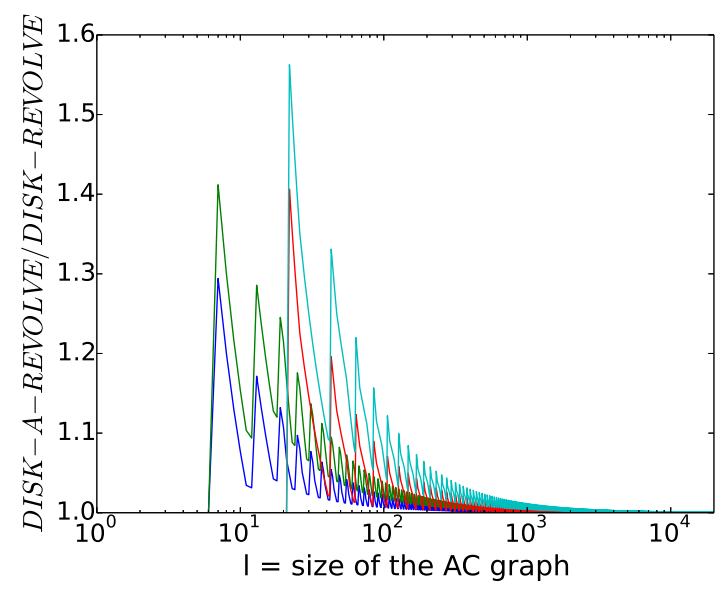

(a) $c_{m}=5$

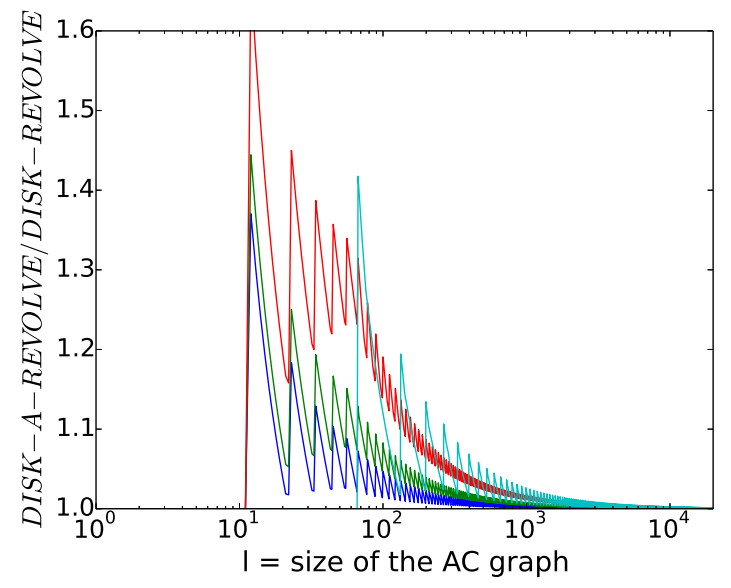

(b) $c_{m}=10$

Figure 5.: Makespan ratio of Disk-A-Revolve $\left(l, c_{m}, w_{d}, r_{d}\right) / \operatorname{Disk-Revolve}\left(l, c_{m}, w_{d}, r_{d}\right)$ as a function of $l$.

Figure $5 \mathrm{a}$ and $5 \mathrm{~b}$ depicts the time overhead of DisK-A-Revolve over the optimal sequence DISK-REVOLVE as a function of $l$. One can see that the asymptotical optimality is attained very quickly (for Adjoint Computation chains of length 1000). As in the offline case, the ratio increases with $w_{d}$ and $r_{d}$. Note that as it is, the online algorithm is not suited for small instances of the problem (small values of $l$ ), indeed, we focused on 
an asymptotically optimal algorithm. However we expect that mixing our asymptotical algorithm with existing online algorithms [9, 11] could improve those cases.

Overall, both $\operatorname{PDS}\left(1, c_{m}, w_{d}, r_{d}\right)$ and DISK-A-REvolve offer good and efficient algorithms to compute an optimal solution to large Adjoint Computation problems.

\section{Conclusion}

In this paper, we have designed optimal algorithms for the hierarchical adjoint computation, with two types of storage location: a limited one with no reading and writing costs and an unlimited one with non-zero access times. We improved the time complexity of the optimal dynamic program introduced in our previous work [1]: by showing that the optimal solution is weakly periodic, we were able to construct an algorithm that returns an optimal schedule for the problem with constant overhead (compared to the quadratic time-complexity of the original optimal algorithm [1]). Furthermore, we also developed asymptotic optimal algorithms that need almost no precomputation. Finally, we provided an asymptotical optimal algorithm for the online problem (when the chain size is not known before-hand).

Modern large-scale cluster are subject to failures that can occur at any time during the computation and lead to a memory flush. The algorithm introduced in this paper establishes a solid foundation to study the impact of memory failures on the performance of hierarchical adjoint computation.

\section{Acknowledgements}

The authors would like to thank Paul Hovland for introducing them to the problem and the idea of a periodic schedule. They would further like to thank Yves Robert and Paul Hovland for constructive conversations. Finally, the authors are extremely grateful to Andrea Walther for a very thorough proofread of this paper.

\section{References}

[1] G. Aupy, J. Herrmann, P. Hovland, and Y. Robert, Optimal multistage algorithm for adjoint computation, Research report RR-8721, INRIA, Apr. 2015, available at http://gaupy.org

[2] R. Giering and T. Kaminski, Recomputations in reverse mode AD, in Automatic Differentiation: From Simulation to Optimization, G. Corliss, C. Faure, A. Griewank, L. Hascoët, and U. Naumann, eds., chap. 33, Computer and Information Science, Springer, New York, 2002, pp. 283-291, Available at http://www .springer.de/cgi-bin/search_book.pl?isbn=0-387-95305-1

[3] A. Griewank, Achieving logarithmic growth of temporal and spatial complexity in reverse automatic differentiation, Optimization Methods and software 1 (1992), pp. 35-54.

[4] A. Griewank and A. Walther, Algorithm 799: Revolve: an implementation of checkpointing for the reverse or adjoint mode of computational differentiation, ACM Transactions on Mathematical Software (TOMS) 26 (2000), pp. 19-45.

[5] M. Hinze and J. Sternberg, A-revolve: an adaptive memory-reduced procedure for calculating adjoints; with an application to computing adjoints of the instationary navier-stokes system, Optimization Methods and Software 20 (2005), pp. 645-663.

[6] R.R. Phelps, Convex functions, monotone operators and differentiability, 1364, Springer Science \& Business Media, 1993.

[7] N.O. SLOANE, Online encyclopaedia of integer sequences, Published electronically at http://www.oeis.org. Accessed July 2015. (2015). 
[8] P. Stumm and A. Walther, Multistage approaches for optimal offline checkpointing, SIAM Journal on Scientific Computing 31 (2009), pp. 1946-1967.

[9] P. Stumm and A. Walther, New algorithms for optimal online checkpointing, SIAM Journal on Scientific Computing 32 (2010), pp. 836-854.

[10] A. Walther, Program reversal schedules for single-and multi-processor machines, Ph.D. diss., $\mathrm{PhD}$ thesis, Institute of Scientific Computing, Technical University Dresden, Germany, 1999.

[11] Q. Wang, P. Moin, and G. Iaccarino, Minimal repetition dynamic checkpointing algorithm for unsteady adjoint calculation, SIAM Journal on Scientific Computing 31 (2009), pp. 2549-2567. 